Bryn Mawr College

Scholarship, Research, and Creative Work at Bryn Mawr

College

2017

\title{
Solid Solid Phase Transitions and tert-Butyl and Methyl Group Rotation in an Organic Solid: X-ray Diffractometry, Differential Scanning Calorimetry, and Solid-State H-1 Nuclear Spin Relaxation
}

Peter A. Beckmann

Bryn Mawr College, pbeckman@brynmawr.edu

Andrew R. McGhie

Arnold L. Rheingold

Gilbert J. Sloan

Steven T. Szewczyk

Let us know how access to this document benefits you.

Follow this and additional works at: http://repository.brynmawr.edu/physics_pubs

Part of the Physics Commons

\section{Custom Citation}

Beckmann, P.A., McGhie, A.R., Rheingold, A.L., Sloan, G.J., Szewczyh, S.T. "Solid Solid Phase Transitions and tert-Butyl and Methyl Group Rotation in an Organic Solid: X-ray Diffractometry, Differential Scanning Calorimetry, and Solid-State H-1 Nuclear Spin Relaxation." Journal of Physical Chemistry A, 121.33 (2017): 6220-6230.

This paper is posted at Scholarship, Research, and Creative Work at Bryn Mawr College. http://repository.brynmawr.edu/physics_pubs/96 For more information, please contact repository@brynmawr.edu. 


\section{Solid-solid Phase Transitions and $t$-Butyl and Methyl Group Rotation in an Organic Solid: X-ray Diffractometry, Differential Scanning Calorimetry, and Solid State ${ }^{1} \mathbf{H}$ Nuclear Spin Relaxation}

Peter A. Beckmann, ${ }^{* \dagger}$ Andrew R. McGhie, ${ }^{\S}$ Arnold L. Rheingold, ${ }^{\ddagger}$ Gilbert J. Sloan, ${ }^{\S}$ and Steven T. Szewczyk ${ }^{ \pm}$

${ }^{\dagger}$ Department of Physics, Bryn Mawr College, 101 North Merion Ave., Bryn Mawr, Pennsylvania 19010-2899, USA.

${ }^{\S}$ Laboratory for the Research on the Structure of Matter, University of Pennsylvania, 3231 Walnut Street, Philadelphia, Pennsylvania 19104-6272, USA.

${ }^{\ddagger}$ Department of Chemistry and Biochemistry, University of California, San Diego, 5128 Urey Hall, 9500 Gilman Dr., La Jolla, California 92093-0358, USA.

${ }^{ \pm}$Department of Materials Science and Engineering, School of Engineering and Applied Science, University of Pennsylvania, 3231 Walnut Street, Philadelphia, Pennsylvania 19104-6272, USA.

Journal of Physical Chemistry A 2017121 6220-6230.

ABSTRACT: Using solid state ${ }^{1} \mathrm{H}$ nuclear magnetic resonance (NMR) spin-lattice relaxation experiments, we have investigated the effects of several solid-solid phase transitions on $t$-butyl group and methyl group rotation in solid 1,3,5-tri-t-butylbenzene. The goal is to relate the dynamics of the $t$-butyl groups and their constituent methyl groups to properties of the solid determined using single-crystal X-ray diffraction and differential scanning calorimetry (DSC). On cooling, the DSC experiments see a first-order, solid-solid phase transition at either $268 \mathrm{~K}$ or 155 $\mathrm{K}$ (but not both) depending on thermal history. The $155 \mathrm{~K}$ transition (on cooling) is identified by single-crystal X-ray diffraction to be one from a monoclinic phase (above $155 \mathrm{~K}$ ) where the $t$-butyl groups are disordered (that is, with a rotational six-fold intermolecular potential dominating) to a triclinic phase (below $155 \mathrm{~K}$ ) where the $t$-butyl groups are ordered (that is, with a rotational threefold intermolecular potential dominating). This transition shows very different DSC scans when both a $5 \mathrm{mg}$ polycrystalline sample and a $19 \mathrm{mg}$ powder sample are used. The ${ }^{1} \mathrm{H}$ spin-lattice relaxation experiments with a much larger $0.7 \mathrm{~g}$ sample are very complicated and, depending on thermal history, can show hysteresis effects over many hours and over very large temperature ranges. In the high-temperature monoclinic phase, the $t$-butyl groups rotate with NMR activation energies (closely related to rotational barriers) in the $17-23 \mathrm{~kJ} \mathrm{~mol}^{-1}$ range and the constituent 
methyl groups rotate with NMR activation energies in the $7-12 \mathrm{~kJ} \mathrm{~mol}^{-1}$ range. In the lowtemperature triclinic phase, the rotations of the $t$-butyl groups and their methyl group in the aromatic plane are quenched (on the NMR time scale). The two out-of-plane methyl groups in the $t$-butyl groups are rotating with activation energies in the $5-11 \mathrm{~kJ} \mathrm{~mol}^{-1}$ range.

\section{INTRODUCTION}

Solid state ${ }^{1} \mathrm{H}$ nuclear magnetic resonance (NMR) spin-lattice relaxation experiments ${ }^{1}$ are helpful in modeling the motion of $t$-butyl groups $\left[\mathrm{C}\left(\mathrm{CH}_{3}\right)_{3}\right]$ and their constituent methyl groups $\left(\mathrm{CH}_{3}\right){ }^{2-12}$ In turn, the parameters that characterize these kinds of rotations contribute to the understanding of the angular anisotropies (for the rotations) in the intramolecular and intermolecular potentials in the solid state. ${ }^{3,13}$ In these kinds of van der Waals molecular solids, composed of small covalently bonded organic molecules with $t$-butyl groups, the molecules usually keep their isolated-molecule structure in the solid state except perhaps for the orientations of the $t$-butyl groups. Here we report and model the unusual dependence on thermal history of the temperature dependence of solid state ${ }^{1} \mathrm{H}$ NMR relaxation measurements in 1,3,5-tri-t-butylbenzene (TTB), a molecule of which is shown in Fig. 1. Differential scanning calorimetry (DSC) measurements reported here find several solid-solid phase transitions that depend on the thermal history in very complicated ways. Singlecrystal X-ray diffraction experiments see one of these transitions in a manner that is consistent with the DSC measurements. These DSC and single-crystal X-ray results go a long way to explaining the very complicated solid state ${ }^{1} \mathrm{H}$ NMR relaxation measurements. The solid state ${ }^{1} \mathrm{H}$ spin-lattice relaxation results from a previous report, ${ }^{14}$ when the solid-solid phase transitions were not known, are confirmed and added to the results reported here. The results reported in a brief conference proceeding, ${ }^{15}$ again, when the phase transitions were not known, are also confirmed and added to the results reported here.

\section{ם 2. METHODS AND RESULTS}

2.1. Sample Preparation. A sample of 1,3,5-tri-t-butylbenzene (TTB) used in the recent experiments was obtained from Sigma Aldrich (97\% mp 340-345 K) and purified by crystallization from the melt using the Bridgman method $^{16}$ in the year 2014. This sample is called sample A. Two other samples (B and C) were recrystallized from methanol in about the year 1980. Samples A and $\mathbf{B}$ were unsealed as discussed in Section 2.4. Sample $\mathbf{C}$, following several freeze- 
pump-thaw cycles, was sealed. Sample $\mathbf{C}$ had been melted and solidified many times (before being sealed) in the freeze-pump-thaw process. Because there are several solid-solid phase transitions in TTB (depending on thermal history), in what follows it is important to distinguish between the very small single crystals used in the single-crystal X-ray diffraction experiments (see Table 1), a $19 \mathrm{mg}$ powder sample used in the DSC experiments, a $5 \mathrm{mg}$ polycrystalline sample used in the DSC experiments, and the approximately $0.7 \mathrm{~g}$ samples used in the solid state ${ }^{1} \mathrm{H}$ NMR relaxation experiments. The mass and morphology differences, along with thermal history effects discussed below, completely trump the differences in the manner in which the samples were purified and originally prepared.

2.2 Single-crystal X-ray Diffraction. A room temperature crystal structure of TTB has been previously published. ${ }^{17}$ This was nearly 40 years ago, the solid-solid phase transitions were not known at the time, and the X-ray diffraction technique has improved considerably in the last four decades. As such, we have repeated the measurements. A single crystal of TTB taken from sample A was mounted on a Hampton CryoLoop with Paratone-N oil and data were collected with a Bruker D8 diffractometer using an Ultra rotating-anode generator (Mo) equipped with a highefficiency multi-layer, double-bounce monochromator. All data were collected with $1.0 \mathrm{sec} / 1.0^{\mathrm{O}}$ correlated scans. Structure solution and subsequent refinement used various components of the SHELXTL software package distributed by the Bruker Corporation (G. Sheldrick, Bruker-AXS, Madison, Wisconsin, USA).

Structures were determined at $175 \mathrm{~K}$ (on reducing temperature from room temperature) and at $100 \mathrm{~K}$ (again, on reducing temperature). The crystal data and structural refinement parameters are reported in Table 1 and the two structures are shown in Figs. 2 and 3. At $175 \mathrm{~K}$ the structure is monoclinic (as was also found in the older room-temperature study ${ }^{17}$ ) and the $t$-butyl groups are disordered as explained further in the caption to Fig. 3 (as was also found in the older roomtemperature study ${ }^{17}$ ). On reducing temperature, many crystals consistently shattered at approximately $155 \mathrm{~K}$, strongly suggesting a first-order structural phase transition at this temperature. A structure, indicated in Fig. 3, was determined at $100 \mathrm{~K}$ with a very small crystal (31 x $15 \times 11 \mathrm{~m}^{3}$; see Table 1), indicating a triclinic structure with ordered (i.e., single equilibrium position) $t$-butyl groups as shown in Figs. 1 and 2.

2.3 Differential Scanning Calorimetry. Differential scanning calorimetry (DSC) was performed with sample A on a TA Instruments Q2000 to characterize thermal transitions. 
Temperature and enthalpy calibrations were made using indium and baseline corrections were determined from sapphire standards. DSC data were collected at a heating and cooling rate of 5 $\mathrm{K} / \mathrm{min}$ under a helium purge.

Fig. 4 (a) and (b) show a series of cooling and heating curves with a $19 \mathrm{mg}$ powder sample beginning at $302 \mathrm{~K}$ after standing overnight at $302 \mathrm{~K}$. A "powder" in this context is a finely ground sample with crystallites having linear dimensions typically in the relatively small range of 1-5 $\mu \mathrm{m}$. There are solid-solid phase transitions at (A) $155 \mathrm{~K}$, (B) $179 \mathrm{~K}$, (C) $255 \mathrm{~K}$, (D) $268 \mathrm{~K}$, and (E) 295 $\mathrm{K}$ labeled A through E in Fig. 4 (a) but they appear and disappear depending on thermal history. The sample was cooled and heated for three cycles. On the first cooling from $302 \mathrm{~K}$, [blue scan labeled 1 in Figs. 4 (a) and (b)], there is one exothermic phase transition at $155 \mathrm{~K}$ [blue peak labeled A in Fig. 4 (a)] with a latent heat of $16 \mathrm{~J} / \mathrm{g}$. This corresponds to the monoclinic-to-triclinic transition found in the single-crystal X-ray diffraction experiments. This transition is shown on an expanded temperature scale in Fig. 5. There are no additional transitions down to $98 \mathrm{~K}$. The baseline for this blue scan in Fig. 4 (a) and (b) is the same as that shown in yellow. Then the temperature is increased from $98 \mathrm{~K}$. The scan is labeled 2 and is green in Fig. 4 (a) and (b). First, there is an endothermic transition at $179 \mathrm{~K}$ (green peak B) with a latent heat of $17 \mathrm{~J} / \mathrm{g}$. This may be the reverse transition found at $155 \mathrm{~K}$ (blue peak A) on cooling but with a $24 \mathrm{~K}$ hysteresis. Then there is an exothermic transition at $255 \mathrm{~K}$ (green peak C) with a latent heat of $17 \mathrm{~J} / \mathrm{g}$. Because this transition is exothermic on heating, the higher temperature phase is more stable than the one below it. On continuing to increase temperature, there is an additional endothermic transition at $295 \mathrm{~K}$ [green peak E, more visible in part (b) of Fig. 4] with a latent heat of $21 \mathrm{~J} / \mathrm{g}$. The baseline for this green scan is the same as that for the black scan. Then the temperature is reversed and the sample cooled before this $295 \mathrm{~K}$ transition (E) is complete. [See the green scan 2 to the brown scan 3 connection at $298 \mathrm{~K}$ in Fig 4 (b).]

Now, on cooling (brown scan 3), the sample undergoes an exothermic transition at $268 \mathrm{~K}$ [brown peak D in Fig. 4 (a)]. This $268 \mathrm{~K}$ transition was not present in the initial scan (blue scan 1). It may be the reverse transition of the $295 \mathrm{~K}$ transition $\mathrm{E}$ (which, in this case, had not gone to completion) with a $27 \mathrm{~K}$ hysteresis. [This $268 \mathrm{~K}$ transition (on cooling) does not appear if the sample is melted and recooled.] On further cooling (brown scan 3 with the same baseline as the yellow scan), there are no additional transitions down to $98 \mathrm{~K}$. That is to say, the transition at 155 $\mathrm{K}$ does not occur. Then, on heating from $98 \mathrm{~K}$ (purple scan 4) transitions B (179 K) and C (255 K) 
do not occur. The purple scan baseline is the same as the black scan baseline in Fig. 4 (a) and (b). Transition E at $295 \mathrm{~K}$ does occur and this time is taken to completion. [See the purple (4)-toyellow (5) scan connection at $303 \mathrm{~K}$ in Fig. 4 (b).] The temperature is then lowered to $98 \mathrm{~K}$ (yellow scan 5) and raised (black scan 6). The result is identical to scans 3 (brown) and 4 (purple). So, it doesn't matter whether transition $\mathrm{E}$ at $295 \mathrm{~K}$ is completed or not.

This all means that when the sample is at room temperature for ten hours or more and cooled to $98 \mathrm{~K}$ through the $155 \mathrm{~K}$ transition, there are 4 stable phases on increasing temperature from $98 \mathrm{~K}$ : one below $155 \mathrm{~K}$, one above $179 \mathrm{~K}$, one above $255 \mathrm{~K}$, and one above $295 \mathrm{~K}$. On cooling again (without melting) there is only one phase transition at $268 \mathrm{~K}$ and then on heating again there is only one transition at $295 \mathrm{~K}$. The black scan 6 was taken to the melting point. TTB melts at $346 \mathrm{~K}$ [Fig. 4 (a)] with a latent heat of $53 \mathrm{~J} / \mathrm{g}$. On subsequent cooling after melting there is one transition at $155 \mathrm{~K}$ and the results of the six scans repeat themselves. This means that melting and then cooling immediately, has the same effect as sitting at $302 \mathrm{~K}$ for many hours. The nature of the three phase transitions at 255,268 , and $295 \mathrm{~K}$ remains to be determined since the X-ray structure, including the disorder in the $t$-butyl groups, reported at $175 \mathrm{~K}$ and at room temperature ${ }^{17}$ are the same.

Fig. 5 shows the same $155 \mathrm{~K}$ monoclinic-to-triclinic transition on decreasing temperature (from being at room temperature overnight) [blue transition A in Fig. 4 (a)] for the $19 \mathrm{mg}$ powder sample on an expanded scale. It also shows the same transition (from being at room temperature overnight) for a $4.7 \mathrm{mg}$ polycrystalline sample. Whereas the powder sample used in Figs. 4 and 5 involved crystallites with linear dimensions typically in the 1-5 $\mu \mathrm{m}$ range, this polycrystalline sample was directly taken from sample A prepared from the melt using the Bridgman ${ }^{16}$ method and was not "powdered." The crystallites will have a greater range of crystallite sizes. The morphology of this $4.7 \mathrm{mg}$ sample, then, was the same as one of the samples used in the NMR relaxation experiments presented in Sections $2.4,2.5$, and 2.6 (which used approximately $0.7 \mathrm{~g}$ ). Fig 5 shows the crystallites transforming at different temperatures according to their size. The sharp peak at $151 \mathrm{~K}$, for example, would be for a relatively large crystallite. This result is important in understanding the NMR relaxation experiments.

\subsection{Solid State ${ }^{1} \mathbf{H}$ Spin-lattice Relaxation Measurements: General Features.}

Relaxation measurements in 1,3,5-tri-t-butylbenzene (TTB) have been going on (on and off) in our laboratory at Bryn Mawr College for 36 years. ${ }^{14,15}$ This is the third report. The solid-solid phase 
transitions presented in Sections 2.2 and 2.3 were only discovered in the year 2014. For the newly reported data, solid state ${ }^{1} \mathrm{H}$ spin-lattice relaxation measurements were performed between the years 1995 and 2010 at $53.0 \mathrm{MHz}$ and between the years 2014 and 2016 at 8.50, 22.5, and 53.0 MHz using an inversion recovery pulse sequence. ${ }^{1,18}$ These experiments used samples $\mathbf{A}, \mathbf{B}$, and $\mathbf{C}$ (see Section 2.1) with different thermal histories as discussed below in Sections 2.5, 2.6, and 2.7.

The NMR line is very broad as expected. ${ }^{18}$ At the lowest temperatures where all rotations are slow on the NMR time scale the rigid-lattice line width $\left(\Delta v_{\text {FWHM }}\right.$ where FWHM means full width at half maximum) is approximately $45 \mathrm{kHz}$ corresponding to a spin-spin relaxation time $T_{2}$ of approximately $7 \mu \mathrm{s}$. [For a Lorentzian, $\Delta v_{\mathrm{FWHM}}=1 /\left(\pi T_{2}\right)$ but the actual line for a polycrystalline sample is a convolution of a Gaussian and a Lorentzian ${ }^{18}$ so these numbers are approximate.] Even at the highest temperatures the line only motionally narrows by approximately a factor of two. The time dependence of the ${ }^{1} \mathrm{H}$ magnetization $M(t)$ following a very short high power $\pi$-pulse perturbation that inverts the whole NMR line can be monitored with a very short $\pi / 2$ pulse that also affects the whole relaxing NMR line. Monitoring $M(t)$ can be done in several ways. One can integrate over various regions of the NMR line or the whole line or one can integrate over various regions of the free induction decay ${ }^{18}$ taking care to start well after the amplifier recovery. It doesn't matter; all procedures give the same magnetization recovery curves $M(t)$. This is a consequence of a strongly coupled ${ }^{1} \mathrm{H}-{ }^{1} \mathrm{H}$ spin-spin dipolar system. Fourier transforming must be done with care when the resulting lines are very wide and we usually choose to integrate a region of the free induction decay which involves fewer steps of data manipulation and is less prone to systematic error.

Temperature was controlled with a homemade cold nitrogen gas flow system and temperature was measured with homemade, silver-soldered, copper-constantan thermocouples that are calibrated to four secondary temperature standards every few years. (The calibration never changes.) Following a temperature change it took approximately 15 minutes for the temperature at the site of the thermocouple to stop changing. We then waited another 30 minutes before performing the next relaxation experiment. The approximately $0.7 \mathrm{~g}$ samples are contained in 6 $\mathrm{mm}$ outer-diameter tubes. The sample portion of the tubes are approximately $20 \mathrm{~mm}$ long, of which $15 \mathrm{~mm}$ is inside the $15 \mathrm{~mm}$-long NMR coil. In samples $\mathbf{A}$ and $\mathbf{B}$, the thermocouple is imbedded in $3 \mathrm{~mm}$ of the sample, $2 \mathrm{~mm}$ from the end of the NMR coil. This means that sample $\mathbf{A}$, prepared in the year 2014 was open to the atmosphere via a small hole for two years during the 
recent experiments and that sample B, prepared in the year 1980, was open to the atmosphere for 36 years when used in recent experiments. In the sealed sample $\mathbf{C}$, the thermocouple is taped to the outside of the sealed glass tube $2 \mathrm{~mm}$ outside the coil. Feedback and ground loop noise from the thermocouple is controlled by using an isolated battery operated microvoltmeter to measure the thermocouple voltage. Absolute temperature (from day to day) was measured to an accuracy of \pm 2 $\mathrm{K}$ and temperature differences during a single day's set of experiments were monitored to an accuracy of $\pm 0.3 \mathrm{~K}$. In what follows in Sections 2.5, 2.6, and 2.7, the NMR relaxation results were the same in samples $\mathbf{A}, \mathbf{B}$, and $\mathbf{C}$ regardless of when they were performed. The results depend only on the thermal history of the sample. So, for example, if sample $\mathbf{B}$ absorbed water from the atmosphere over 36 years, it had absolutely no effect whatsoever on the relaxation results.

2.5. Exponential Relaxation: Thermal Preparation 1. For the results presented and discussed in this section, the samples were thermally recycled three times between $77 \mathrm{~K}$ and room temperature (293-299 K) over a period of one hour (referred to as "thrice-cycled"). In this case the relaxation was always exponential and strictly reproducible regardless of subsequent temperature changes (increasing and decreasing) in the range 85-303 K throughout a 16 hour period. The time dependence of the ${ }^{1} \mathrm{H}$ magnetization $M(t)$ was fitted to $M(t)=M(\infty)+[M(0)-M(\infty)][\exp (-R t)]$. This is a three-parameter fit; the (unique) spin-lattice relaxation rate $R$ (the inverse of the spin-lattice relaxation time $\left.T_{1}\right)$, the equilibrium magnetization $M(\infty)$, and the initial perturbed magnetization $M(0)$. Ideally, $M(0)=-M(\infty)$ for a perturbation $\pi$-pulse sequence but the pulse sequences are not perfect and if $M(0)$ is not taken as an independent fitting parameter, systematic errors in $R$ can result.

$R$ versus temperature $T$ is shown in Fig. 6 at $8.50 \mathrm{MHz}$ (Ref. 14 using sample $\mathbf{B}$ and three data points from this work using sample $\mathbf{A}$ ), $22.5 \mathrm{MHz}$ (this work using samples $\mathbf{A}, \mathbf{B}$, and $\mathbf{C}$ ), and 53.0 $\mathrm{MHz}$ (Ref. 14 using sample $\mathbf{B}$ and three data points from this work using sample A). Which sample is used plays absolutely no role whatsoever, nor does its thermal history prior to the three recycles matter for at least 16 hours after the three recycles. Since temperature was changed by both small and large amounts between 85 and $303 \mathrm{~K}$ over the 10-16 hours each day, it can be concluded that a 45 minute wait after the temperature was changed was adequate for the approximately $0.7 \mathrm{~g}$ sample to achieve thermal equilibrium. Given the more complicated results presented below in Sections 2.6 and 2.7, this strongly implies that for these thrice-cycled experiments with a $0.7 \mathrm{~g}$ sample there were no phase transitions. The manner in which the entire $R$ 
versus $T$ curve scales with NMR frequency (Fig. 6) also suggests that no phase transitions are occurring in the sample.

The exponential $R$ versus $T$ data in Fig. 6 can be reasonably well-fitted to a wellestablished model ${ }^{4,5}$ based on the Bloch-Redfield-Wangsness model of spin-lattice relaxation ${ }^{19-22}$ as first extended to $t$-butyl plus methyl group rotation by Dunn and McDowell. ${ }^{23}$ This model is very robust and in this case we assume all three $t$-butyl groups in the molecule are dynamically equivalent. There are seven terms in the expression for $R$; one for the $t$-butyl group rotation, one each for the rotations of the three methyl groups, and one each for the superimposed rotations of each methyl group and the $t$-butyl group. Each term is of the form $R_{\mathrm{k}}=A_{\mathrm{k}} J\left(\omega, \tau_{\mathrm{k}}\right)[\mathrm{k}=1$ to 7$]$ and here we choose the simplest random-motion spectral density with a unique correlation time $\tau_{\mathrm{k}}$; $J\left(\omega, \tau_{\mathrm{k}}\right)=2 \tau_{\mathrm{k}} /\left(1+\omega^{2} \tau_{\mathrm{k}}^{2}\right){ }^{5,18,24}$ (In Section 2.7 we will also choose a different spectral density.) Here, $\tau_{\mathrm{k}}$ is an NMR correlation time (for the k-th rotation) that can be taken as the mean time between hops in a semiclassical methyl and $t$-butyl group hopping process. (We do not need to consider quantum mechanical tunneling at these high temperatures. ${ }^{25-34}$ ) Four of the $\tau_{\mathrm{k}}$ involve the four rotors in a $t$-butyl group and the other three $\tau_{\mathrm{k}}$ are the superposition terms. Fig. 6 shows the total relaxation rate at the three NMR frequencies; $\omega /(2 \pi)=8.50,22.5$, and $53.0 \mathrm{MHz}$ for the single fit. Fig. 6 also shows the contributions to the relaxation rate at $53.0 \mathrm{MHz}$ of the $t$-butyl group rotation and the rotations of three methyl groups alone plus methyl group $+t$-butyl group superimposed rotations. There are two adjustable parameters for each of the four individual rotations. ${ }^{4}$ These are a preexponential factor ${ }^{4,5,35-37} \tau_{\propto \circ \mathrm{k}}$ and an NMR activation energy ${ }^{4,5,38-40} E_{\mathrm{k}}$ in an Arrhenius relation $\tau_{\mathrm{k}}=\tau_{\triangleright \mathrm{k}} \exp \left(E_{\mathrm{k}} / k T\right)$. The seven values of $A_{\mathrm{k}}$ in the seven $R_{\mathrm{k}}=A_{\mathrm{k}} J\left(\omega, \tau_{\mathrm{k}}\right)$ can all be related to a single adjustable parameter ${ }^{4,5}$ so in addition to the eight adjustable parameters characterizing the correlation times, there is an additional single parameter characterizing the strength of the spin-spin interactions. ${ }^{4,5}$ This parameter is indicative of the number of rotors contributing to the relaxation and for this thrice-cycled $R$ versus $T$ data (thermal preparation 1) all three $t$-butyl groups and their constituent methyl groups in TTB are reorienting on the NMR time scale in various temperature regions between 85 and $303 \mathrm{~K}$. For the single fit in Fig. 6, the $t$-butyl group activation energy is $E=20 \mathrm{~kJ} \mathrm{~mol}^{-1}$ and the three methyl groups have activation energies of $E=10,10$, and $7.5 \mathrm{~kJ} \mathrm{~mol}^{-1}$. There are other sets of NMR activation energies that would be equally successful but the $t$-butyl group activation energy will be in the $17-23 \mathrm{~kJ} \mathrm{~mol}^{-1}$ range and the three methyl group activation energies will all be in the $7-12 \mathrm{~kJ} \mathrm{~mol}^{-1}$ range. The important point here is 
that all $t$-butyl groups and their constituent methyl groups are contributing to the observed relaxation in various temperature regions between 85 and $303 \mathrm{~K}$ and the parameters that characterize these rotation are similar to the parameters found for similar planar organic van der Waals molecular solids. ${ }^{2-5,10-12,41-43}$ There are significant systematic differences between the fitted and observed relaxation rates for the fit shown, especially at $53.0 \mathrm{MHz}$ in the $160-220 \mathrm{~K}$ range in Fig. 6. Other sets of four activation energies within the ranges given above will shift these differences between theory and experiment to other temperature ranges and to another NMR frequency, but they cannot be eliminated. One can let the three $t$-butyl groups be only slightly different (as a consequence of intermolecular interactions) and this would increase the number of adjustable parameters from 9 to 27 . Then one could fit a dog's hind leg. The point to be made is that all twelve rotors in every molecule are involved in the relaxation process and all three $t$-butyl groups are approximately dynamically equivalent.

When compared with the relaxation rate data discussed below in Sections 2.6 and 2.7, these results are consistent with the sample being in the high-temperature monoclinic phase where the $t$ butyl groups are disordered. This means that a six-fold rotational potential dominates and (quantum mechanically speaking) one can think of a $t$-butyl group as having six half-methyl groups. The potential responsible for the $17-23 \mathrm{~kJ} \mathrm{~mol}^{-1}$ barrier for $t$-butyl group rotation must be intermolecular in origin since in the isolated molecule, the $t$-butyl group rotational barrier will be in the 2-4 kJ mol${ }^{-1}$ range. ${ }^{3}$ Finally, we note that given the near-oblate average shape of the molecules (i.e., the shape of the time-averaged electron density) one might expect whole-molecule rotation to occur, at least about one axis. But this is definitely not occurring on the NMR time scale (or faster) in which case the NMR relaxation results would be very different. We cannot rule out the possibility that whole-molecule rotation occurs very slowly (on the NMR time scale).

2.6. Nonexponential Relaxation: Thermal Preparation 2. A single day's set of eleven experiments at $22.5 \mathrm{MHz}$ (performed in the year 2016 using sample A) takes into account the manner in which the phase transitions appeared and disappeared in the differential scanning calorimetry experiments. Having been at room temperature for several weeks, the $0.7 \mathrm{~g}$ sample was placed in the NMR probe which had been preset at $110 \mathrm{~K}$, well below the $155 \mathrm{~K}$ phase transition. It was left in for ten minutes and then removed and then left for 20 minutes at $295 \mathrm{~K}$. It was then put back into the NMR coil which was still at $110 \mathrm{~K}$ and, in a single day, eleven measurements made between $93 \mathrm{~K}$ and $190 \mathrm{~K}$. We refer to this as thermal preparation 2. The 
relaxation was nonexponential below $140 \mathrm{~K}$ and exponential above $140 \mathrm{~K}$. When the relaxation was not exponential to within experimental uncertainty, the initial relaxation rate $R_{\mathrm{S}}$ [using $M(t)$ values for only short times $t$ ] was determined using a technique outlined in detail elsewhere. ${ }^{44} R_{\mathrm{S}}$ is a convenient parameter to characterize nonexponential relaxation, in part, because this is the parameter that is amenable to theoretical interpretation if cross correlations among the three ${ }^{1} \mathrm{H}$ spins in a methyl group are responsible for the nonexponential relaxation and the methyl group rotation axis is not reorienting on the NMR time scale. ${ }^{44-48}$ This effect manifests itself predominantly near the relaxation rate maximum $(\omega \tau \sim 1)$ and in the high-temperature shortcorrelation time limit $(\omega \tau<<1)$ and much less so in the low-temperature long-correlation time limit $(\omega \tau \gg>1) .{ }^{44-48}$ In the current case, however, the relaxation is highly nonexponential in lowtemperature limit. As such, this phenomenon is probably not the cause of the nonexponential relaxation. $R_{\mathrm{S}}$ versus $T$ for eight measurements between 93 and $132 \mathrm{~K}$ (over a period of eight hours) is indicated by red circles in Fig. 7. Three additional measurements were then made at 146, 165 , and $190 \mathrm{~K}$ over an additional period of five hours. The relaxation for these three measurements was exponential and $R$ versus $T$ is indicated by the three red squares in Fig. 7.

For the experiments at $22.5 \mathrm{MHz}$, in addition to determining $R_{\mathrm{S}}$, the entire nonexponential $M(t)$ below $140 \mathrm{~K}$ was well fitted with a five-parameter double exponential $M(t)=M_{1}(t)+M_{2}(t)=\left\{M_{1}(\infty)+\left[M_{1}(0)-M_{1}(\infty)\right]\left[\exp \left(-R_{1} t\right)\right]\right\}+\left\{M_{2}(\infty)+\left[M_{2}(0)-M_{2}(\infty)\right]\left[\exp \left(-R_{2} t\right)\right]\right\}$. The five fitting parameters can be taken to be $R_{1}, R_{2}, M_{1}(\infty), M_{2}(\infty)$, and $M(0)$ where $M(0)=M_{1}(0)+M_{2}(0)$ but where not both $M_{1}(0)$ and $M_{2}(0)$ are independent. For double exponential relaxation, it is convenient to replace the two absolute equilibrium magnetizations $M_{1}(\infty)$ and $M_{2}(\infty)$ with the fractional equilibrium magnetizations $\phi_{1}=M_{1}(\infty) /\left[M_{1}(\infty)+M_{2}(\infty)\right]$ and $\phi_{2}=M_{2}(\infty) /\left[M_{1}(\infty)+M_{2}(\infty)\right]$. An example of $M(t)$ described by a double exponential is shown in Fig. 8. The observation of double exponential relaxation suggests that there are two types of regions (clusters of molecules) and that the regions are large enough such that ${ }^{1} \mathrm{H}-{ }^{1} \mathrm{H}$ spin diffusion, which can operate over distances involving many molecules, is not effective between them. These two sets of regions, which need not be contiguous and are likely spread throughout the sample, have magnetizations characterized by $M_{1}(t)$ and $M_{2}(t) . R_{1}$ and $R_{2}$ versus $T$ for the eight nonexponential relaxation measurements are indicated by green inverted triangles and green upright triangles in Fig. 7. The fractional magnetizations ranged from $\phi_{1} / \phi_{2}=30 \% / 70 \%$ to $50 \% / 50 \%$. 
For these data in Fig. 7, $R_{\mathrm{S}} \sim R_{1}$ (green downward pointing triangles) below $140 \mathrm{~K}$ have a similar temperature dependence as the exponential $R$ values in the thrice-cycled samples (unfilled diamonds). We suggest that these $R_{1} \sim R_{\mathrm{S}} \sim R$ values characterize that part of the sample in the hightemperature monoclinic phase where all $t$-butyl groups and their constituent methyl groups are reorienting on the NMR time scale in various temperature regions as indicated in Fig. 6 and as described in Section 2.5. The green upward pointing triangles indicate $R_{2}$ values smaller than the $R_{1}$ values by more than a factor of five. Fig. 8 shows an example where $R_{1} / R_{2}=6.5$. We suggest these $R_{2}$ values are indicative of the low-temperature triclinic phase where the $t$-butyl groups are ordered, resulting from a dominant contribution from an intermolecular three-fold component to the potential. The dynamical model for the intramolecular motion corresponding to the $R_{2}$ values is discussed in the next section.

2.7. Nonexponential Relaxation: Thermal Preparations 3 and 4. Thermal preparations 3 and 4 , though very different, gave the same highly nonexponential relaxation results that were very different than the nonexponential relaxation results for thermal preparation 2 discussed in the previous section. For preparation 3, the sample had been at room temperature for days or weeks and then inserted into the NMR probe. For preparation 4, the sample, having been at room temperature for days or weeks was put in liquid nitrogen $(77 \mathrm{~K})$ for many hours and then inserted into the NMR probe. In both cases, the probe was preset at the desired temperature between 105 and $140 \mathrm{~K}$. Subsequent experiments during the five days of experiments [three with thermal preparation 3 and two with thermal preparation 4 (one in liquid nitrogen for 12 hours and the other for 50 hours)] were performed by increasing temperature. The relaxation was always nonexponential below $150 \mathrm{~K}$, sometimes exponential and sometimes nonexponential between 150 and $205 \mathrm{~K}$ and always exponential above $205 \mathrm{~K}$. $R_{\mathrm{S}}$ versus $T$ data at $53.0 \mathrm{MHz}$ are presented with red circles in Fig. 9. The scatter in $R_{\mathrm{S}}$ values is not due to there being two different thermal preparations. The scatter is the same for both preparations which is why we present them all with the same symbols. Red squares indicate that the relaxation was exponential. Three sets of these 53.0 MHz data in Fig. 9 are newly reported here (using both thermal preparations) and two sets (one for each thermal preparation) are from our previous report in the year $1992 .{ }^{15}$ The old and new data are the same and again, it didn't matter which sample was used. Thermal history prior to the thermal preparation played no role. When the relaxation is highly nonexponential, $R_{\mathrm{S}}$, though 
determined using a precise procedure, ${ }^{44}$ has large uncertainties. The uncertainties in the $R_{\mathrm{S}}$ values in Fig. 9 (and Fig. 7) are taken to be $\pm 20 \%$.

The $R_{\mathrm{S}}$ values for the highly nonexponential relaxation data from preparations 3 and 4 in Fig. 9 are significantly smaller than the $R_{1} \sim R_{\mathrm{S}} \sim R$ values for thermal preparation 2 in Fig. 7. They are closer in value to the $R_{2}$ values in Fig. 7 . This suggests there is very little of the hightemperature monoclinic phase present in the sample.

The Bloch-Redfield-Wangsness model of spin-lattice relaxation ${ }^{19-22}$ is again used to give at least some idea of the dynamics to which these $R_{\mathrm{S}}$ values correspond. $R_{\mathrm{S}}=A J(\omega, \tau)$ only now we only need a single term. The fit corresponds to a dynamical model where all $t$-butyl group rotation is quenched (that is, static on the NMR time scale). (If $t$-butyl groups are rotating on the NMR time scale, the relaxation is much more exponential and $R$ is significantly larger ${ }^{2,4,5,42}$ as indicated by the unfilled squares in Fig. 9). In addition, two-thirds of the methyl groups are rotating on the NMR time scale and rotation for one-third of the methyl groups is also quenched. In the lowtemperature triclinic phase, the orientation of the $t$-butyl groups is such that one methyl group is in or very near the plane of the aromatic ring (Figs. 1 and 2). If the $t$-butyl groups are static on the NMR time scale it is likely that this in-plane methyl group is also static on the NMR time scale since this methyl group and the $t$-butyl group usually have the same activation energy for this geometry because the in-plane methyl group will "gear" around the aromatic plane as the $t$-butyl group rotates. ${ }^{3,4,42,49}$ The two out-of-plane rotating methyl groups have the same correlation time $\tau=\tau_{\infty} \exp (E / k T)$. We only use the data below $200 \mathrm{~K}$ for this fit since at higher temperatures the sample is always in the high-temperature monoclinic phase and the relaxation is exponential. As such, only the low-temperature limit $(\omega \tau \gg>1) R_{\mathrm{S}}$ versus $T$ data is available. The high-temperature, short correlation time limit $(\omega \tau<<1)$ is not available. This means that the spectral density $J(\omega, \tau)$ cannot be unambiguously determined. ${ }^{24}$ For the fit shown through the red circles in Fig. 9, the random motion spectral density ${ }^{24} J(\omega, \tau)=2 \tau /\left(1+\omega^{2} \tau^{2}\right)$ predicts $E=6 \pm 1 \mathrm{~kJ} \mathrm{~mol}^{-1}$ for the two rotating methyl groups. This is very small for the two out-of-plane methyl groups in a $t$-butyl group. The intra-t-butyl group interaction provides a barrier of $15-19 \mathrm{~kJ} \mathrm{~mol}^{-1}{ }^{3}$ This would mean that the intermolecular component of the potential would be out-of-phase with the intra-t-butyl component in the sense that it subtracts from it. This does happen. ${ }^{50}$ If a Davidson-Cole spectral density ${ }^{24,51-53}$ $J(\omega, \tau)=(2 / \omega)[\sin \{\operatorname{carctan}(\omega \tau)\}] /\left[\left(1+\omega^{2} \tau^{2}\right)^{\varepsilon / 2}\right]$ is used for the same fit in Fig. 9, then $\varepsilon E=6 \pm 1 \mathrm{~kJ}$ 
$\mathrm{mol}^{-1}$ and it is not possible to separate the two parameters $\varepsilon$ and $E$ without data in the hightemperature regime $(\omega \tau<<1)$. We have discussed the meaning of $\varepsilon<1$ extensively: ${ }^{51,52}$ it implies a distribution of activation energies (rotational barriers) with $E$ being the upper-limit cutoff. When $\varepsilon=1$, the Davidson-Cole spectral density reduces to the unique $\tau$ random-motion spectral density. ${ }^{24}$ The parameter $\varepsilon$ is typically $0.6<\varepsilon<1$ which means that $E$ is in the range $5-11 \mathrm{~kJ} \mathrm{~mol}^{-1}$.

We suggest that these highly nonexponential relaxation measurements, characterized by $R_{\mathrm{S}}$ versus $T$ in Fig. 9 result from the sample being predominantly in the low-temperature triclinic phase but with a large range of crystallite sizes with many being very small. Indeed, if the crystallites are small enough, there might be regions of the sample that would lose their crystalline nature and be better described as amorphous (a glass). This would result in a distribution of activation energies since the out-of-plane methyl groups in the $t$-butyl groups would see a variation of intermolecular environments. One must distinguish between a distribution of methyl group activation energies and highly nonexponential relaxation. One can have a wide distribution of activation energies but still have exponential relaxation if the sample is homogeneous enough and ${ }^{1} \mathrm{H}-{ }^{1} \mathrm{H}$ spin diffusion maintains a single spin temperature throughout the sample. But in this case, the highly nonexponential relaxation suggests that the clusters of molecules or the very small crystallites do not communicate effectively with each other via ${ }^{1} \mathrm{H}-{ }^{1} \mathrm{H}$ spin-spin interactions.

\section{a. DISCUSSION AND CONCLUSIONS}

We have investigated solid 1,3,5-tri-t-butylbenzene (TTB) using single-crystal X-ray diffraction, solid state ${ }^{1} \mathrm{H}$ spin-lattice relaxation, and differential scanning calorimetry (DSC). The molecule is highly symmetric and this can result in several first-order, solid-solid phase transitions with large hysteresis regions. On an initial cooling from room temperature, there is a monoclinic-to-triclinic transition at $155 \mathrm{~K}$ and on heating from $98 \mathrm{~K}$, this transition occurs at $179 \mathrm{~K}$. But this transition disappears on subsequent coolings, then heatings, so long as the sample is not melted or left at room temperature for long periods of time. There is also a $255 \mathrm{~K}$ transition found on heating a first time from $98 \mathrm{~K}$ but not on subsequent coolings then heatings. Then there is a transition at $295 \mathrm{~K}$ only found on heating from $98 \mathrm{~K}$ but in this case on every heating. This transition occurs at $268 \mathrm{~K}$ on cooling but only on a second and subsequent coolings and in this case the $155 \mathrm{~K}$ transition is not observed. TTB melts at $346 \mathrm{~K}$. This behavior means that thermal history plays an important role and the behavior of small samples $(19 \mathrm{mg})$ used in the differential scanning calorimetry 
experiments may be very different from the thermal history of large samples $(0.7 \mathrm{~g})$ used in the NMR relaxation experiments. The thermal history effects in the large samples can last at least tens of hours.

This report is the third study of solid state ${ }^{1} \mathrm{H}$ spin-lattice relaxation in 1,3,5-tri-tbutylbenzene (TTB), the previous two being in the year $1984^{14}$ and in the year $1992 .{ }^{15}$ The goal is to try to understand the relationship between the solid state ${ }^{1} \mathrm{H}$ nuclear magnetic resonance (NMR) spin-lattice relaxation parameters that characterize the rotations of the $t$-butyl groups and their constituent methyl groups and the state of a (relatively) large $(0.7 \mathrm{~g})$ macroscopic sample of the solid. The several solid-solid phase transitions whose appearance and disappearance depend strongly on thermal history makes the above sought-after relationship both more complicated, more interesting, and potentially more informative concerning the role of the intermolecular interactions.

Solid state ${ }^{1} \mathrm{H}$ spin-lattice relaxation experiments can provide information on the dynamics of intramolecular moieties. This information, in turn, can provide information on the anisotropies of intramolecular and intermolecular interactions. These investigations involve the littleunderstood mesoscopic scale for molecular solids. Much is known about the properties of individual molecules (the microscopic scale) from gas phase experiments and from electronic structure calculations. ${ }^{3,48,51,54,55}$ In addition, much is known about the macroscopic properties (physical and thermodynamic) of solids. But there is still much to be learned about the relationship between the structure and the dynamics on the mesoscopic scale; that is, tens to hundreds of molecules. ${ }^{1} \mathrm{H}$ relaxation NMR exploits the long range nature of the ${ }^{1} \mathrm{H}-{ }^{1} \mathrm{H}$ spin-spin interactions to investigate this scale. The downside of solid state ${ }^{1} \mathrm{H}$ relaxation NMR is that relatively large macroscopic samples are usually required and the information concerning the dynamics of intramolecular moieties in a polycrystalline sample is arrived at by models that assume large ensembles of molecules (that are not necessarily contiguous) are dynamically equivalent, or nearly so.

In this study of 1,3,5-tri-t-butylbenzene, we find that the high-temperature solid-solid phase transitions at 255, 268, and $295 \mathrm{~K}$ (found by differential thermal calorimetry in a $19 \mathrm{mg}$ sample) have no effect on the ${ }^{1} \mathrm{H}$ NMR relaxation in a $0.7 \mathrm{~g}$ sample. Since single-crystal X-ray diffractometry finds both the molecular and crystal structure above and below these transitions is the same, the nature of these transition is yet to be determined. The monoclinic-to-triclinic 
transition at $155 \mathrm{~K}$ on cooling (found by both single-crystal X-ray diffractometry and by differential scanning calorimetry) has a significant effect on the NMR relaxation and this effect depends on thermal history in the $0.7 \mathrm{~g}$ NMR sample. One thermal preparation (Section 2.5) leaves the sample in the high-temperature monoclinic phase even well below $155 \mathrm{~K}$. In this case we find that the ${ }^{1} \mathrm{H}$ spin-lattice relaxation is exponential and that all the $t$-butyl groups and their constituent methyl groups are rotating on the NMR time scale with NMR activation energies (closely related to rotational barriers ${ }^{39,40}$ ) in the $17-23 \mathrm{~kJ} \mathrm{~mol}^{-1}$ range and that the methyl groups rotate with NMR activation energies in the $7-12 \mathrm{~kJ} \mathrm{~mol}^{-1}$ range.

A second thermal preparation with the $0.7 \mathrm{~g}$ NMR samples (Section 2.6) leaves the sample in a mixture of the two phases below $155 \mathrm{~K}$ which is stable for long periods of time. Two additional quite different thermal preparations (Section 2.7) with the $0.7 \mathrm{~g}$ NMR samples leave the sample in the low-temperature triclinic phase but in a state where many crystallites are very small and some regions may be so small as to lose their crystallinity and be in an amorphous (glass) state. Again this highly nonequilibrium state persist for many hours. In this state, the $t$-butyl groups and the methyl group in the plane of the aromatic ring are static on the NMR time scale. The two out-of-plane methyl groups are rotating on the NMR time scale with NMR activation energies in the $5-11 \mathrm{~kJ} \mathrm{~mol}^{-1}$ range.

It would be useful to perform electronic structure calculations ${ }^{3,48,51,54-56}$ in clusters of molecules based on the X-ray structure in both the high-temperature monoclinic and lowtemperature triclinic phases. These studies can calculate rotational barrier heights for both $t$-butyl group and methyl group rotation, at least in slightly larger molecules with rigid (on the NMR time scale) backbones. ${ }^{3}$ For clusters of 1,3,5-tri- $t$-butylbenzene, where the $t$-butyl groups are a significant fraction of the volume of the molecule (see Fig. 1) and where their rotational barriers are dominated by intermolecular interactions, the cluster would have to be quite large (probably $5^{3}-6^{3}=125-216$ molecules) and structural relaxation of all the atoms on at least several molecules near a central molecule would have to be taken into account as a methyl and/or a $t$-butyl group on a central molecule of the cluster is rotated (both one at a time and together). The energy of the entire cluster has to be calculated when a specific rotor on a central molecule is in a rotational ground state and this entire cluster energy has to be calculated again when this same rotor is in a transition state. (First, these two states have to be found.) Then the two (very large) energies have 
to be subtracted to obtain a (relatively small) barrier height. The energy landscape would be manydimensional because of all the rotations involved and the calculations would be very challenging.

\section{aUTHOR INFORMATION}

Corresponding Author

*Email: pbeckman@brynmawr.edu. Phone: +011-6105265358

\section{口 ACKNOWLEDGMENTS}

ARM and GJS acknowledge support from NSF Grant DMR-1120901. PAB acknowledges the support, for several decades, of the Bryn Mawr College Director of Science Services, Richard Willard, and the Science Services Chief Instrument Maker, Rob Cunningham. The Bryn Mawr Solid State NMR lab could not function without their continued support.

\section{- REFERENCES}

${ }^{1}$ Kimmich, R. NMR Tomography, Diffusometry, Relaxometry; Springer-Verlag: Berlin, 1997.

${ }^{2}$ Beckmann, P. A.; Moore, C. E.; Rheingold, A. L. Methyl and $t$-Butyl Group Rotation in a Molecular Solid: ${ }^{1} \mathrm{H}$ NMR Spin-lattice Relaxation and X-ray Diffraction. Phys. Chem. Chem. Phys. 2016, 18, 1720-1726.

${ }^{3}$ Wang X.; Mallory, F. B.; Mallory, C. W.; Odhner, H. R.; Beckmann, P. A. Solid State ${ }^{1}$ H Spinlattice Relaxation and Isolated-molecule and Cluster Electronic Structure Calculations in Organic Molecular Solids: The Relationship Between Structure and Methyl Group and $t$-Butyl Group Rotation. J. Chem. Phys. 2014, 140, 194304, 1-15.

${ }^{4}$ Beckmann, P. A.; Buser, C. A.; Gullifer, K.; Mallory, F. B.; Mallory, C. W.; Rossi, G. M.; Rheingold, A. L. Methyl and $t$-Butyl Group Reorientation in Planar Aromatic Solids: Lowfrequency NMR Relaxometry and X-ray Diffraction. J. Chem. Phys. 2003, 118, 11129-11138.

${ }^{5}$ Beckmann, P. A.; Hill, A. I.; Kohler, E. B.; Yu, H. Nuclear-spin Relaxation in Molecular Solids with Reorienting Methyl and $t$-Butyl Groups: The Spectral Density and the State of the Solid. Phys. Rev. B 1988, 38, 11098-11111.

${ }^{6}$ Goc, R. Simulation of the NMR Second Moment as a Function of Temperature in the Presence of Molecular Motion. Application to $\left(\mathrm{CH}_{3}\right) \mathrm{ONBH}_{3}$. Zeit. für Naturfor. A 2002, 57, 29-35.

${ }^{7}$ Gruwel, M. L. H. Orientation Dependence of ${ }^{2} \mathrm{H}$ Nuclear Spin Relaxation in Trimethyl- $\left[{ }^{2} \mathrm{H} 9\right]-$ Ammonium Tetraphenylborate. J. Chem. Soc., Faraday Trans. 1991, 87, 1715-1718. 
${ }^{8}$ Szcezesniak, E. Molecular Dynamics and Phase Transitions in Solid 2-Methyl-2-Propanethiol Studied by ${ }^{1}$ H N.M.R. Relaxation. Mol. Phys. 1986, 58, 551-560.

${ }^{9}$ Konieczka, R.; Pajak, Z. Pulsed and Continuous Wave Nuclear Magnetic Resonance Study of the Complex Molecular Motion in $t$-Butylammonium Bromide. J. Chem. Soc., Faraday Trans. 2 1981, 77, 2041-2047.

${ }^{10}$ Pettitt, B. A.; Lewis, J. S.; Wasylishen, R. E.; Danchura, W.; Tomchuk, E.; Bock, E. A. Deuterium NMR Study of Orientational Order in tertiary-Butyl Bromide. J. Mag. Resonan. 1981, $44,50-517$.

${ }^{11}$ Yamauchi, J.; McDowell, C. A. Magnetic Relaxation of Solid Ammonia-Boron Trifluoride Complex. J. Chem. Phys. 1981, 75, 577-583.

${ }^{12}$ Yamauchi, J.; McDowell, C. A. NMR Study of Molecular Motion in Some Molecules Containing $t$-Butyl Groups. J. Chem. Phys. 1981, 75, 1051-1059.

${ }^{13}$ Beckmann, P. A.; Rheingold, A. L. ${ }^{1} \mathrm{H}$ and ${ }^{19} \mathrm{~F}$ Spin-lattice Relaxation and $\mathrm{CH}_{3}$ or $\mathrm{CF}_{3}$ Reorientation in Molecular Solids Containing Both H and F Atoms. J. Chem. Phys. 2016, 144, $154308,1-12$.

${ }^{14}$ Albano, A. M.; Beckmann, P. A.; Carrington, M. E.; Fisch, E. E.; Fusco, F. A.; O'Neill, A. E.; Scott, M. E. Nuclear Spin Relaxation Study of Internal Motion in Two Organic Molecular Solids. Phys. Rev. B 1984, 30, 2334-2339.

${ }^{15}$ Beckmann, P. A. Proton Spin Relaxation and Thermal History Effects in Organic Molecular Solids. In Physics and Chemistry of Finite Systems: From Clusters to Crystals, ed. Jena, P.; Khanna, S. N.; Rao, B. K. Kluwer Academic: Netherlands 1992, 1, 357-362.

${ }^{16}$ Goodman, C. H. L. Crystal Growth Theory and Techniques Vol. 1; Plenum: London, 1974.

${ }^{17}$ Saika, T. Crystal and Molecular Structure of 1,3,5-Tri-tert-butylbenzene. Acta. Cryst. 1978, B34, 3649-3653.

${ }^{18}$ Abragam, A. The Principles of Nuclear Magnetism; Oxford University Press: Oxford, UK, 1961.

${ }^{19}$ Bloch, F. Dynamical Theory of Nuclear Induction II. Phys. Rev. 1956, 102, 104-135.

${ }^{20}$ Bloch, F. Generalized Theory of Relaxation. Phys. Rev. 1957, 105, 1206-1222.

${ }^{21}$ Redfield, A. G. The Theory of Relaxation Processes. IBM J. Res. Develop. 1957, 1, 19-31. Reprinted with minor revisions in Advan. Mag. Resonan. 1965, 1, 1-32.

${ }^{22}$ Wangsness, R. K.; Bloch, F. The Dynamical Theory of Nuclear Induction. Phys. Rev. 1953, 89, 728-739. 
${ }^{23}$ Dunn, M. B.; McDowell, C. A. An N.M.R. Study of Molecular Motion in Solid Trimethylaminegallane. Molec. Phys. 1972, 24, 969-978.

${ }^{24}$ Beckmann, P. A. Spectral densities and nuclear spin relaxation in solids. Phys. Rep. 1988, 171 $85-128$.

${ }^{25}$ Clough, S. NMR and Relative Quantum Theory. Sol. State Nuc. Mag. Resonan. 1997, 9, 49-53.

${ }^{26}$ Barlow, M. J.; Clough, S.; Horsewill, A. J.; Mohammed, M. A. Rotational Frequencies of Methyl Group Tunneling. Sol. State Nuc. Mag. Resonan. 1992, 1, 197-204.

${ }^{27}$ Clough, S. Coherent Methyl Dynamics, J. Phys. C: Sol. State Phys. 1994, 202, 256-263.

${ }^{28}$ Clough, S. Quantum Tunnelling and Classical Hopping Motion of Molecules. Physica B 1986, $136,145-149$.

${ }^{29}$ Cavagnat, D.; Clough, S.; Zelaya, F. O. Fast Hopping of Deuterated Methyl Groups. J. Phys. C: Sol. State Phys. 1985, 18, 6457-6462.

${ }^{30}$ Clough, S.; McDonald, P. J.; Zelaya, F. O. The Transition from Free Quantum Tunnelling to Thermally Driven Motion of Methyl Groups. J. Phys. C: Sol. State Phys. 1984, 17, 4413-4420.

${ }^{31}$ Clough, S.; McDonald, P. J. The Correlation of Methyl Tunnelling and Thermally Activation Reorientation: II. J. Phys. C: Sol. State Phys. 1982, 15, L1039-L1042.

${ }^{32}$ Clough, S.; Heidemann, A.; Horsewill, A. J.; Lewis, J. D.; Paley, M. N. J. The Correlation of Methyl Tunnelling and Thermally Activation Reorientation. J. Phys. C: Sol. State Phys. 1981, 14, L525-L529.

${ }^{33}$ Clough, S.; Heidemann, A. The Approach to Classical Dynamics of Reorienting Methyl Groups. J. Phys. C: Sol. State Phys. 1980, 13, 3585-3589.

${ }^{34}$ Stejskal, E. O.; Gutowsky, H. S. Proton Magnetic Resonance of the $\mathrm{CH}_{3}$ Group. IV. Calculation of the Tunneling Frequency and of $\mathrm{T}_{1}$ in Solids. J. Chem. Phys. 1958, 28, 388-396.

${ }^{35}$ Waugh J. S.; Fedin É. I. Determination of Hindered-rotation Barriers in Solids. Soviet Physics Solid State 1963, 4, 1633-1636.

${ }^{36}$ Ferrando, R.; Spadacini, R.; Tommei, G. E.; Mel'nikov, V. I. Numerical Test of Finite-barrier Corrections for the Hopping Rate in the Underdamped Regime. Phys. Rev. E 1995, 51, R1645R1648.

${ }^{37}$ Owen, N. L. in Internal Rotation in Molecules, ed. Orville-Thomas, W. J. Wiley: New York, 1974.

${ }^{38}$ van Putte, E. A. Spin-lattice Relaxation of $\mathrm{CH}_{3}$ and $\mathrm{CH}_{2} \mathrm{D}$ Groups in Some Partially Deuterated Alkanes. J. Mag. Resonan. 1970, 2, 216-225. 
${ }^{39}$ Kowaleski, J.; Liljefors, T. On the Relationship Between the Potential Barrier and the Activation Energy for the Internal Rotation of a Methyl Group. Chem. Phys. Lett. 1979, 64, 170-174.

${ }^{40}$ Edholm, O.; Blomberg, C. Brownian Motion Description of Activation Energies from NMRrelaxation Times for Rotating Molecular Groups. Chem. Phys. 1981, 56, 9-14.

${ }^{41}$ Popa, L. C.; Rheingold, A. L.; Beckmann, P. A. A Proton Spin-lattice Relaxation Rate Study of Methyl and $t$-Butyl Group Reorientation in the Solid State. Sol. State Nuc. Mag. Resonan. 2010, $38,31-35$.

${ }^{42}$ Beckmann, P. A.; Dougherty Jr., W. G.; Kassel, W. S. Methyl and $t$-Butyl Reorientation in an Organic Molecular Solid. Sol. State Nuc. Mag. Resonan. 2009, 36, 86-91.

${ }^{43}$ Wang, X.; Rheingold, A. L.; DiPasquale, A. G.; Mallory, F. B.; Mallory, C. W.; Beckmann, P. A. The Quenching of Isopropyl Group Rotation in van der Waals Molecular Solids. J. Chem. Phys. 2008, 128, 124502, 1-3.

${ }^{44}$ Beckmann P. A.; Schneider E. Methyl Group Rotation, ${ }^{1} \mathrm{H}$ Spin-lattice Relaxation in an Organic Solid, and the Analysis of Nonexponential Relaxation. J. Chem. Phys. 2012, 136, 054508, 1-9.

${ }^{45}$ Runnells L. K. Nuclear Spin-lattice Relaxation in Three-spin Molecules. Phys. Rev. 1964, 134, A28-A36.

${ }^{46}$ Hilt, R. L.; Hubbard, P. S. Nuclear Magnetic Relaxation of Three Spin Systems Undergoing Hindered Rotations. Phys. Rev. 1964, 134, A392-A398.

${ }^{47}$ Beckmann, P. A. Nonexponential ${ }^{1} \mathrm{H}$ Spin-lattice Relaxation and Methyl Group Rotation in Molecular Solids. Sol. State Nuc. Mag. Resonan. 2015, 71, 91-95.

${ }^{48}$ Beckmann, P. A.; Mallory, C. W.; Mallory, F. B.; Rheingold, A. L.; Wang, X. Methoxy and Methyl Group Rotation: Solid State NMR ${ }^{1}$ H Spin-lattice Relaxation, Electronic Structure Calculations, X-ray Diffractometry, and Scanning Electronic Microscopy. ChemPhysChem 2015, $16,1509-1519$.

${ }^{49}$ Beckmann, P. A.; Burbank, K. S.; Lau, M. M. W.; Ree, J. N.; Weber, T. L. Solid State Proton Spin-lattice Relaxation in Four Structurally Related Organic Molecules. Chem. Phys. 2003, 290, 241-250.

${ }^{50}$ Beckmann, P. A.; Al-Hallaq, H. A,; Fry, A. M.; Plofker, A. L.; Roe, B. A.; Weiss, J. A. Solid State Proton Spin Relaxation and Methyl and $t$-Butyl Reorientation. J. Chem. Phys. 1994, 100, 752-753.

${ }^{51}$ Beckmann, P. A.; Conn, K. G.; Mallory, C. W.; Mallory, F. B.; Rheingold, A. L.; Rotkina, L.; Wang, X. Distributions of Methyl Group Rotational Barriers in Polycrystalline Organic Solids. J. Chem. Phys. 2013, 139, 204501, 1-12. 
${ }^{52}$ Beckmann, P. A.; Happersett, L.; Herzog, A. V.; Tong, W. M. Solid State Proton Spin Relaxation in Ethylbenzenes: Methyl Reorientation Barriers and Molecular Structure. J. Chem. Phys. 1991, 95, 828-835.

${ }^{53}$ Davidson, D. W.; Cole, R. H. Dielectric Relaxation in Glycerol, Propylene, and n-Propanol. $J$. Chem. Phys. 1951, 19, 1484-1490.

${ }^{54}$ Wang, X.; Rotkina, L.; Su, H.; Beckmann, P. A. Single-crystal X-ray Diffraction, Isolatedmolecule and Cluster Electronic Structure Calculations, and Scanning Electron Microscopy in an Organic Solid: Models for Intramolecular Motion in 4,4'-Dimethoxybiphenyl. ChemPhysChem 2012, 13, 2082-2089.

${ }^{55}$ Wang, X.; Beckmann, P. A.; Mallory, C. W.; Rheingold, A. L.; DiPasquale, A. G.; Carroll, P. J.; Mallory, F. B. Intramolecular and Intermolecular Contributions to the Barriers for Rotation of Methyl Groups in Crystalline Solids: Electronic Structure Calculations and Solid State NMR Relaxation Measurements. J. Org. Chem. 2011, 76, 5170-5176.

${ }^{56}$ Kohanoff, J. Electronic Structure Calculations for Solids and Molecules: Theory and Computational Methods; Cambridge Univ. Press: Cambridge, UK, 2006 


\section{TOC GRAPHIC}

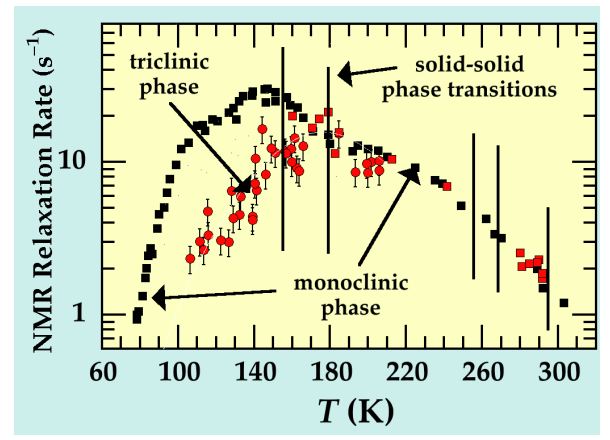


Table 1. Crystal data and structure refinement for 1,3,5-tri-t-butylbenzene; the monoclinic phase at $175 \mathrm{~K}$ and the triclinic phase at $100 \mathrm{~K}$.

\begin{tabular}{|c|c|c|}
\hline CDCC Identification code & 1525485 & 1525484 \\
\hline Empirical formula & $\mathrm{C}_{18} \mathrm{H}_{30}$ & $\mathrm{C}_{18} \mathrm{H}_{30}$ \\
\hline Formula weight & 246.42 & 246.42 \\
\hline Temperature & $175 \mathrm{~K}$ & $100 \mathrm{~K}$ \\
\hline Wavelength & $0.71073 \AA$ & $0.71073 \AA$ \\
\hline Crystal system & monoclinic & triclinic \\
\hline Space group & $\mathrm{C} 2 / \mathrm{c}$ & $\mathrm{P}-1$ \\
\hline \multirow[t]{6}{*}{ Unit cell parameters } & $a=10.1047(8) \AA$ & $a=9.861(2) \AA$ \\
\hline & $b=17.4637(8) \AA$ & $b=10.0803(19) \AA$ \\
\hline & $c=19.8059(10) \AA$ & $c=19.560(4) \AA$ \\
\hline & $\alpha=90^{\circ}$ & $\alpha=90.176(14)^{\circ}$. \\
\hline & $\beta=90.291(4)^{\circ}$ & $\beta=90.198(14)^{\circ}$. \\
\hline & $\gamma=90^{\circ}$ & $\gamma=119.276(11)^{\circ}$. \\
\hline Volume & $3495.0(4) \AA^{3}$ & $1695.9(6) \AA^{3}$ \\
\hline$Z$ & 8 & 4 \\
\hline$Z$ & 1 & 2 \\
\hline Density (calculated) & $0.937 \mathrm{Mg} / \mathrm{m}^{3}$ & $0.965 \mathrm{Mg} / \mathrm{m}^{3}$ \\
\hline Absorption coefficient & $0.052 \mathrm{~mm}^{-1}$ & $0.053 \mathrm{~mm}^{-1}$ \\
\hline $\mathrm{F}(000)$ & 1104 & 552 \\
\hline Crystal size & $0.37 \times 0.34 \times 0.32 \mathrm{~mm}^{3}$ & $0.031 \times 0.015 \times 0.011 \mathrm{~mm}^{3}$ \\
\hline Theta range for data collection & 2.057 to $30.971^{\circ}$ & 1.041 to $25.482^{\circ}$ \\
\hline Index ranges & $-13 \leq h \leq 14,-22 \leq k \leq 25,-27 \leq l \leq 28$ & $-11 \leq h \leq 11,-12 \leq k \leq 12,0 \leq l \leq 23$ \\
\hline Reflections collected & 16209 & 18114 \\
\hline Independent reflections & $5296[R($ int $)=0.0371]$ & $6193[R($ int $)=0.0451]$ \\
\hline Completeness to $q=26.000^{\circ}$ & $99.9 \%$ & $100.0 \%$ \\
\hline Absorption correction & Semi-empirical from equivalents & Semi-empirical from equivalents \\
\hline Max. and min. transmission & 0.9837 and 0.9811 & 1.000 and 0.923 \\
\hline Refinement method & Full-matrix least-squares on $F^{2}$ & Full-matrix least-squares on $F^{2}$ \\
\hline Data / restraints / parameters & $5296 / 0 / 265$ & $6193 / 0 / 344$ \\
\hline Goodness-of-fit on $F^{2}$ & 1.021 & 1.044 \\
\hline Final $R$ indices $[\mathrm{I}>2 \operatorname{sigma}(\mathrm{I})]$ & $R 1=0.0686, w R 2=0.1794$ & $R 1=0.0765, w R 2=0.1661$ \\
\hline$R$ indices (all data) & $R 1=0.1335, w R 2=0.2141$ & $R 1=0.1475, w R 2=0.2025$ \\
\hline Largest diff. peak and hole & 0.198 and -0.154 e $\AA^{-3}$ & 0.282 and $-0.279 e \AA^{-3}$ \\
\hline
\end{tabular}




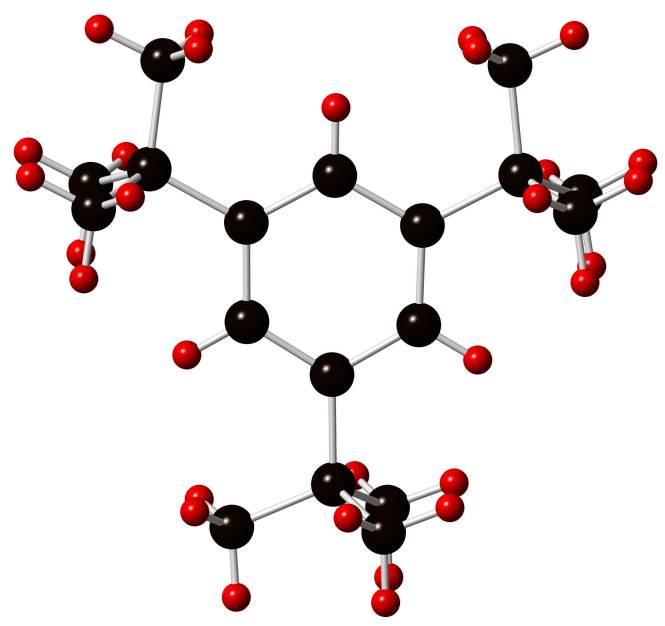

Fig. 1. The molecule 1,3,5-tri-t-butylbenzene (TTB). C atoms are black and $\mathrm{H}$ atoms are red. The structure shown is that of the molecule seen by single-crystal X-ray diffraction at $100 \mathrm{~K}$ in the low-temperature triclinic phase (see Table 1). Although the asymmetric unit is two molecules having slightly different structures, the structures (Fig. 2) appear the same for the orientation and resolution shown here. All three $t$-butyl groups have one methyl group in the plane of the aromatic ring. The bottom $t$-butyl group and the one to the upper left have the same orientation (with respect to the aromatic ring) but the $t$-butyl group to the upper right is rotated by $60^{\circ}$. 


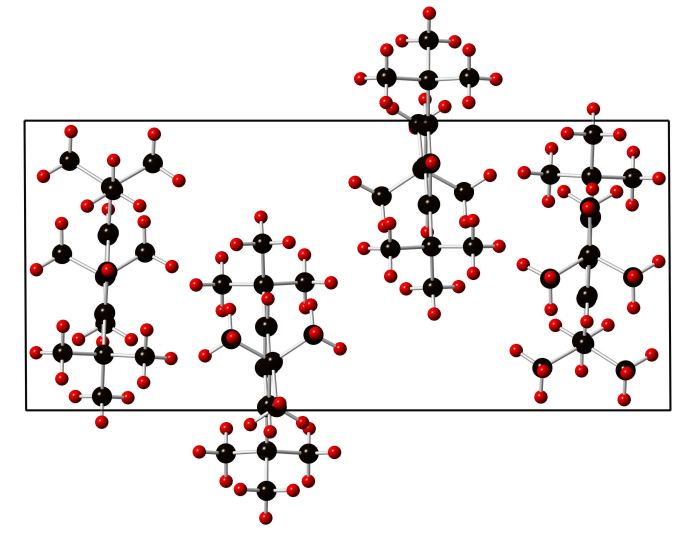

(a)

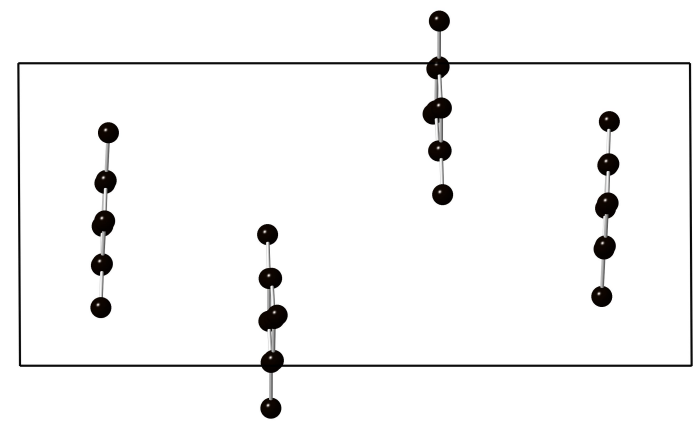

(b)

Fig. 2. The crystal structure of 1,3,5-tri-t-butylbenzene (TTB) in the 100 plane in the lowtemperature triclinic phase as seen by single-crystal X-ray diffraction at $100 \mathrm{~K}$ (see Table 1). C atoms are black and $\mathrm{H}$ atoms are red. (a) Full molecules and (b) with methyl groups deleted leaving only the aromatic ring and quaternary $\mathrm{C}$ atoms. The solid lines are the unit cell. There are four molecules per unit cell as shown $(Z=4)$. Only whole molecules are shown. Where parts of molecules project out of the unit cell, the same parts of other molecules project into the unit cell from the opposite face. The asymmetric unit is two molecules $(Z=2)$; either the two on the left or the two on the right. The molecular structure is shown in Fig. 1 and the difference between the structure of the two molecules in the asymmetric unit is negligible for the orientation shown in Fig. 1. The $t$-butyl groups are ordered and have the orientations discussed in the caption to Fig. 1, implying that a three-fold component of the intermolecular potential dominates. 


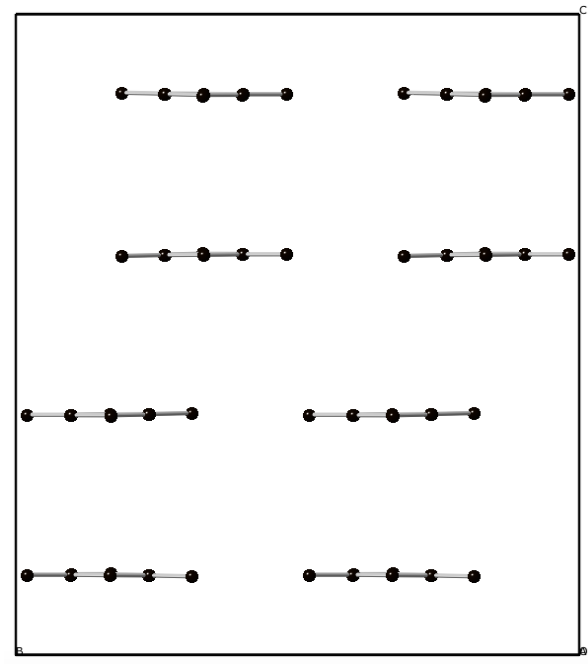

(a)

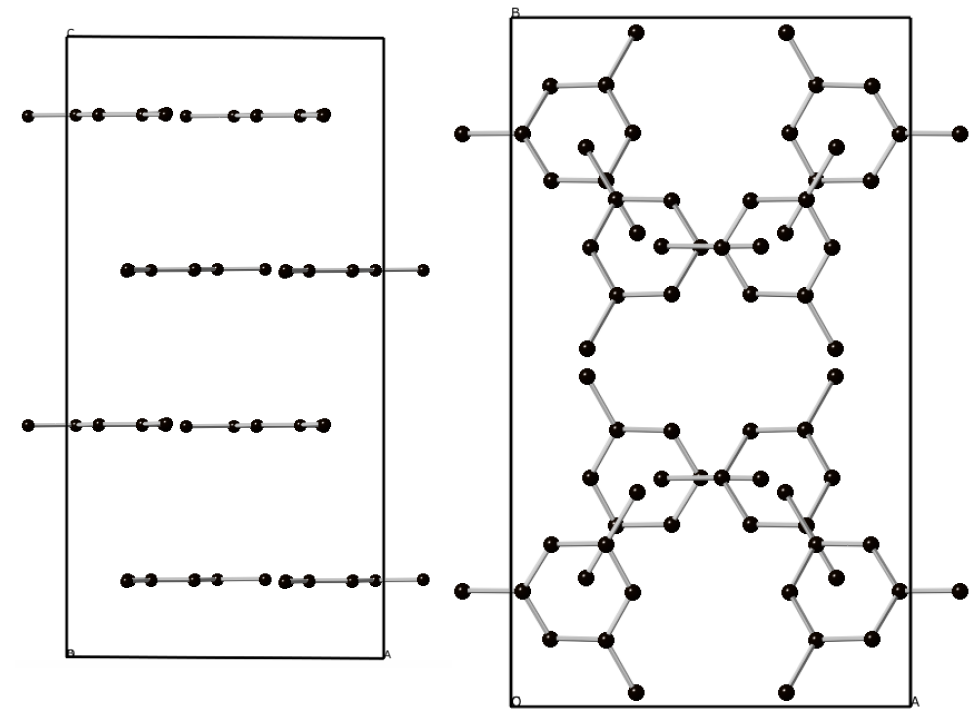

(b) (c)

Fig. 3. The crystal structure of 1,3,5-tri-t-butylbenzene (TTB) in the high-temperature monoclinic phase seen by single-crystal X-ray diffraction. The projections are (a) the (100), (b) the (010), and (c) the (001) planes and the solid lines are the unit cell. The unit cell angles are $90^{\circ}, 90^{\circ}$, and $90.29^{\mathrm{O}}$ so the unit cell (monoclinic) is almost orthorhombic. There are eight molecules per unit cell $(Z=8)$ as shown. Only whole molecules are shown and all methyl groups have been eliminated for clarity, so only aromatic ring and quaternary $\mathrm{C}$ atoms remain. Where parts of molecules project out of the unit cell, the same parts of other molecules project into the unit cell from the opposite face. The asymmetric unit is a single molecule $(Z=1)$. The $t$-butyl groups are disordered meaning that each $t$-butyl group should be thought of as being a linear combination of one orientation (as shown by any one of the three $t$-butyl groups in the molecule shown in Fig. 1) and another rotated by $60^{\mathrm{O}}$. Such $t$-butyl groups can be thought of (quantum mechanically speaking) as having six half-methyl groups. This also means that the dominant component of the intermolecular potential has 6-fold symmetry. This molecular structure would be similar to that found in the isolated molecule (the gas phase) since the dominant intramolecular potential for the $t$-butyl groups (whose magnitude is significantly smaller than the intermolecular potential) is also 6-fold. 


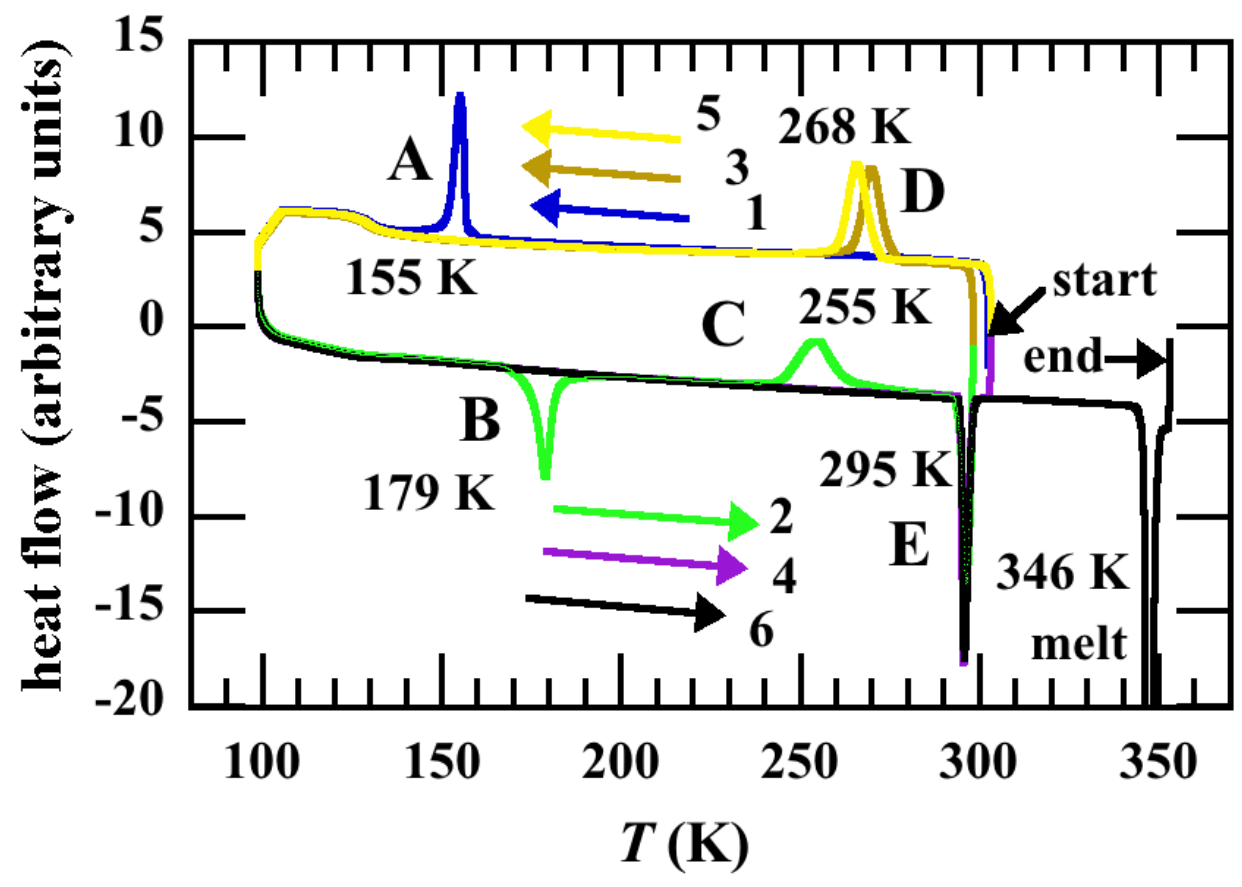

(a)

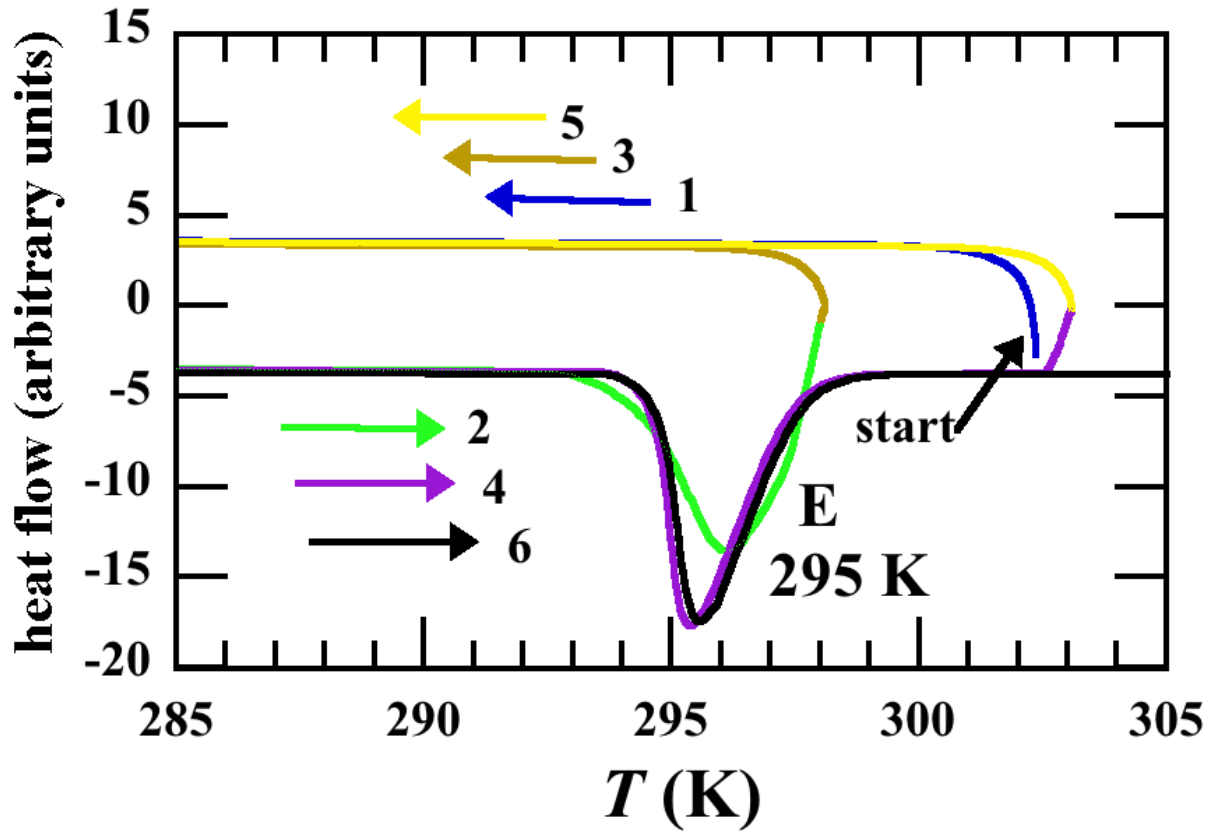

(b) 
Fig. 4. Differential scanning calorimetry (DSC) scans in a $19 \mathrm{mg}$ powder sample of 1,3,5-tri-tbutylbenzene (TTB). (a) shows three cycles of decreasing temperature [scans 1 (blue), 3 (brown), and 5 (yellow)] and increasing temperature [scans 2 (green), 4 (purple), and 6 (black)]. The starting and ending temperatures are indicated. The baselines for scans 1, 3, and 5 (decreasing temperature) are the same (yellow) and the baselines for scans 2, 4, and 6 (increasing temperature) are the same (black). The solid-solid phase transitions are labeled A through $\mathrm{E}$ and the melt is indicated. These scans are discussed in Section 2.3. (b) The region centered around transition E in (a) on an expanded scale. 


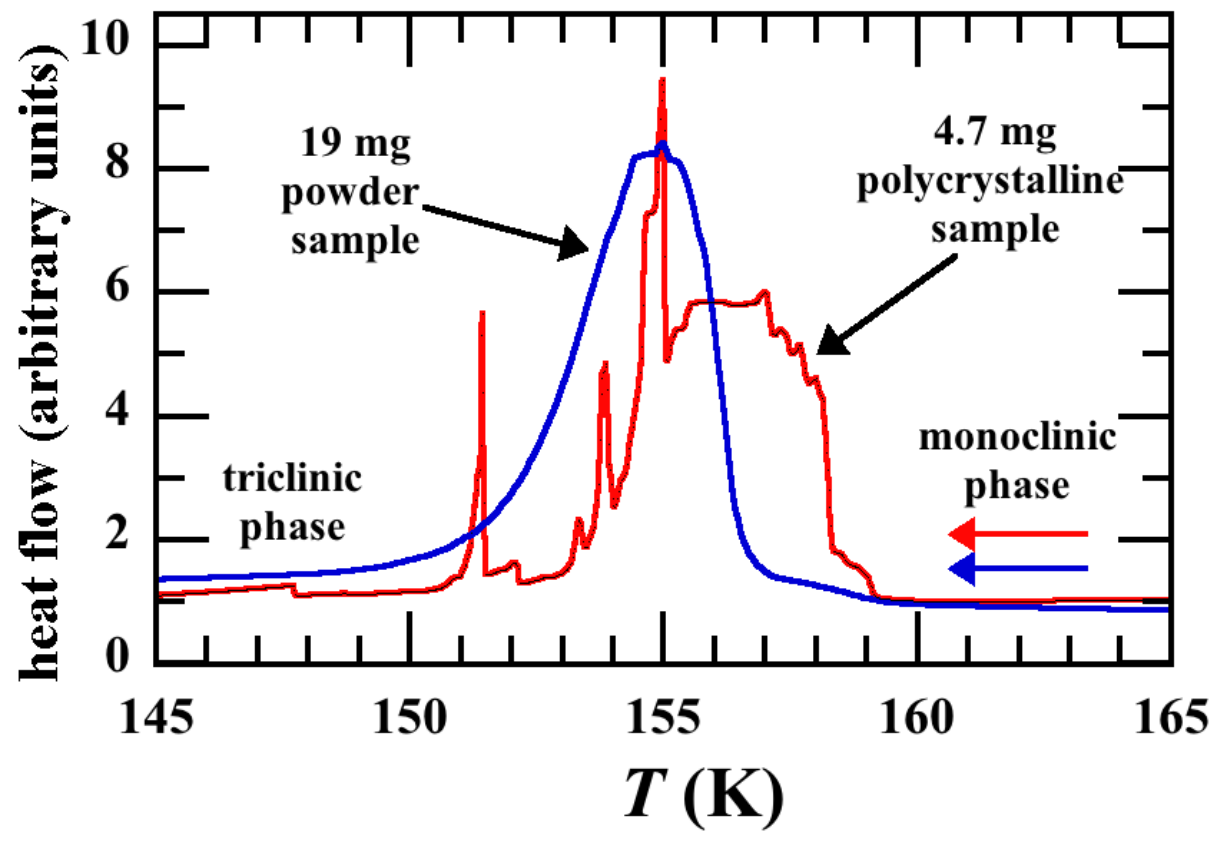

Fig. 5. The $155 \mathrm{~K}$ solid-solid phase transition (on decreasing temperature for a first time from room temperature or following a melt) in 1,3,5-tri-t-butylbenzene (TTB). The smooth blue line in the $19 \mathrm{mg}$ powder sample is the same as the blue transition labeled A in Fig. 4 (a). The red line with several individual peaks is the same transition with the same thermal history in a $4.7 \mathrm{mg}$ polycrystalline sample. The terms 'powder' and 'polycrystalline' are discussed in Section 2.3. In both cases, the scans were $5 \mathrm{~K} / \mathrm{min}$. 


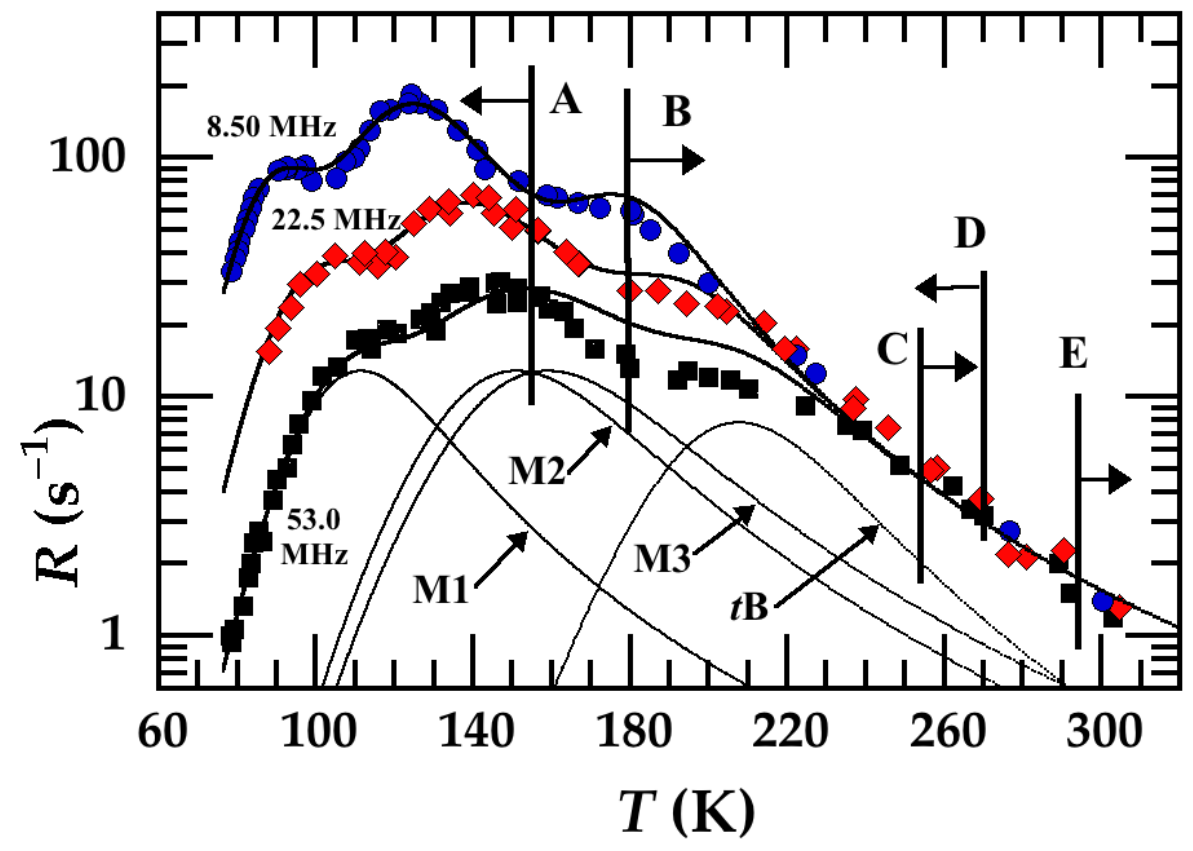

Fig. 6. The ${ }^{1} \mathrm{H}$ spin-lattice exponential relaxation rate $R$ versus temperature $T$ in $0.7 \mathrm{~g}$ samples of 1,3,5-tri-t-butylbenzene (TTB): [8.50 MHz \{ (blue)\} (Ref. 14 and this work), $22.5 \mathrm{MHz}\{$ (red)\} (this work), and 53.0 MHz $\{\mathbf{\square}$ (black)\} (Ref. 14 and this work)]. All samples were recycled three times between 77 and 293-299 K ("thrice cycled") over a one-hour period prior to 6-15 measurements over 10-16 hours. The uncertainty bars are within the size of the symbols. The solid-solid phase transitions labeled by A through E seen (depending on thermal history) by scanning differential calorimetry in a $19 \mathrm{mg}$ powder sample (Fig. 4) are indicated by the black vertical lines with a right-pointing arrow meaning on heating and a left-pointing arrow meaning on cooling. The three solid lines through the data at the three NMR frequencies are a single fit to $R$ versus $T$ (as discussed in Section 2.5) that assumes the phase transitions are not occurring and that all $t$-butyl groups are dynamically equivalent. The four components of the $53.0 \mathrm{MHz}$ fit are shown; $t \mathrm{~B}$ labels the relaxation resulting from the rotation of the $t$-butyl group and M1-M3 each label the sums of the relaxation resulting from the rotation of each methyl group plus the superposition of the rotations of the methyl group and the $t$-butyl group. 


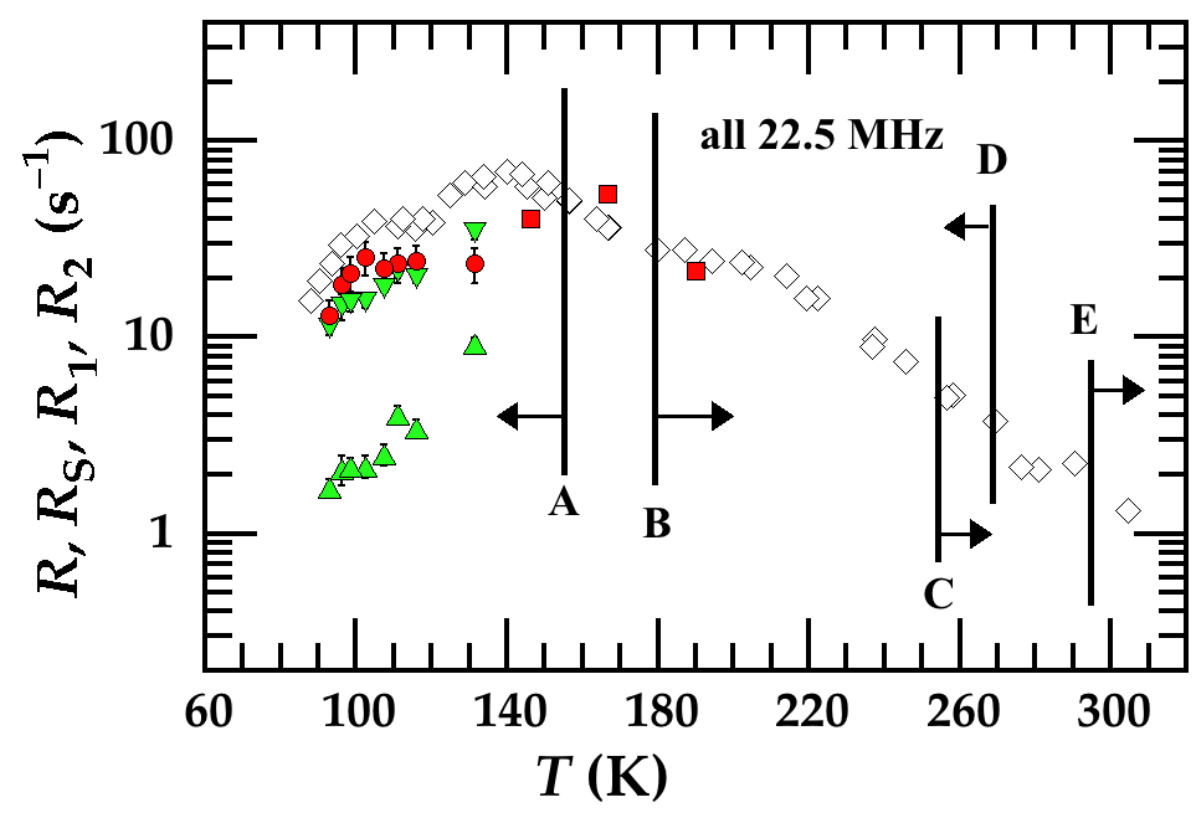

Fig. 7. Various ${ }^{1} \mathrm{H}$ spin-lattice relaxation rates versus temperature $T$ in 1,3,5-tri-t-butylbenzene (TTB) at an NMR frequency of $22.5 \mathrm{MHz} \cdot R_{1}\left[\nabla\right.$ (green)] and $R_{2}[\Delta$ (green)] are the two relaxation rates in a double exponential relaxation process, $R_{\mathrm{S}}[(\mathrm{red})]$ is the initial relaxation rate in a nonexponential relaxation process (from the same data that yielded $R_{1}$ and $R_{2}$ ), and $R$ [ $\square$ (red)] is the relaxation rate in an exponential relaxation process. The complicated thermal preparation (thermal preparation 2) of the sample (followed by the eleven measurements in a single 13-hour day) is discussed in Section 2.5. The fractional equilibrium magnetizations $\phi_{1}$ and $\phi_{2}$ characterizing the double exponential relaxation rates $R_{1}$ and $R_{2}$ shown here range from $\phi_{1} / \phi_{2}=30 \% / 70 \%$ to $50 \% / 50 \%$. The exponential relaxation rate $R[\diamond$ (unfilled diamonds)] in thricecycled samples (thermal preparation 1) shown in Fig. 6 is repeated here for comparison. The solidsolid phase transitions labeled by A through E seen (depending on thermal history) by scanning differential calorimetry (Fig. 4) are indicated by the black vertical lines with a right-pointing arrow meaning on heating and a left-pointing arrow meaning on cooling. 


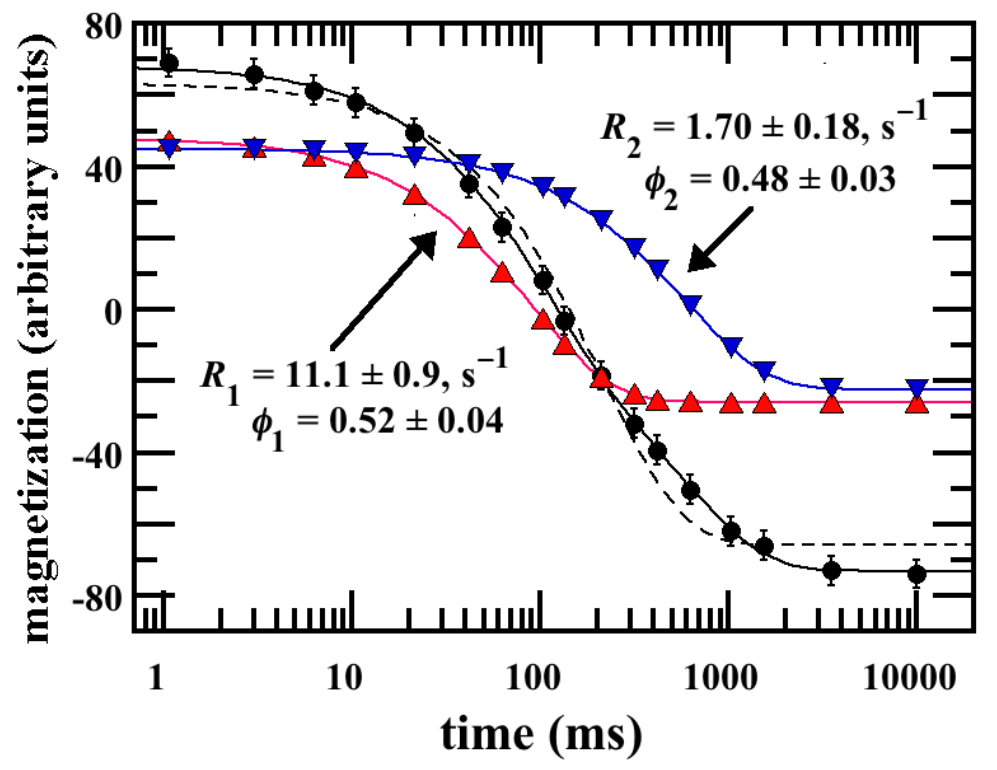

Fig. 8. An example of a double exponential ${ }^{1} \mathrm{H}$ magnetization relaxation curve $M(t)=M_{1}(t)+M_{2}(t)=\left\{M_{1}(\infty)+\left[M_{1}(0)-M_{1}(\infty)\right]\left[\exp \left(-R_{1} t\right)\right]\right\}+\left\{M_{2}(\infty)+\left[M_{2}(0)-M_{2}(\infty)\right]\left[\exp \left(-R_{2} t\right)\right]\right\}$ at $93 \mathrm{~K}$ in 1,3,5-tri-t-butylbenzene (TTB) at an NMR frequency of $22.5 \mathrm{MHz}$ [ (black)]. The component magnetizations $M_{1}(t)$ [ $\boldsymbol{\Delta}$ (red)], and $M_{2}(t)$ [ $\boldsymbol{\nabla}$ (blue)] in the fit are shown. The time between the perturbation and observe pulses is indicated on a logarithmic scale solely to better resolve the relaxation over all four orders of magnitude in time. The five adjustable parameters can be taken to be $R_{1}, R_{2}, M_{1}(\infty), M_{2}(\infty)$, and $M(0)$ where $M(0)=M_{1}(0)+M_{2}(0)$ but not both $M_{1}(0)$ and $M_{2}(0)$ are independent. Here $R_{1}=11.1 \pm 0.9 \mathrm{~s}^{-1}, \phi_{1}=M_{1}(\infty) /\left[M_{1}(\infty)+M_{2}(\infty)\right]=0.52 \pm 0.04, R_{2}=1.70 \pm 0.18$ $\mathrm{s}^{-1}$, and $\phi_{2}=M_{2}(\infty) /\left[M_{1}(\infty)+M_{2}(\infty)\right]=0.48 \pm 0.03$. The $R_{1}$ and $R_{2}$ values are the lowest temperature values (green triangles) in Fig. 7. A fit to a three-parameter single exponential [with adjustable parameters $R, M(\infty)$, and $M(0)$ ] is shown by the dashed line. 


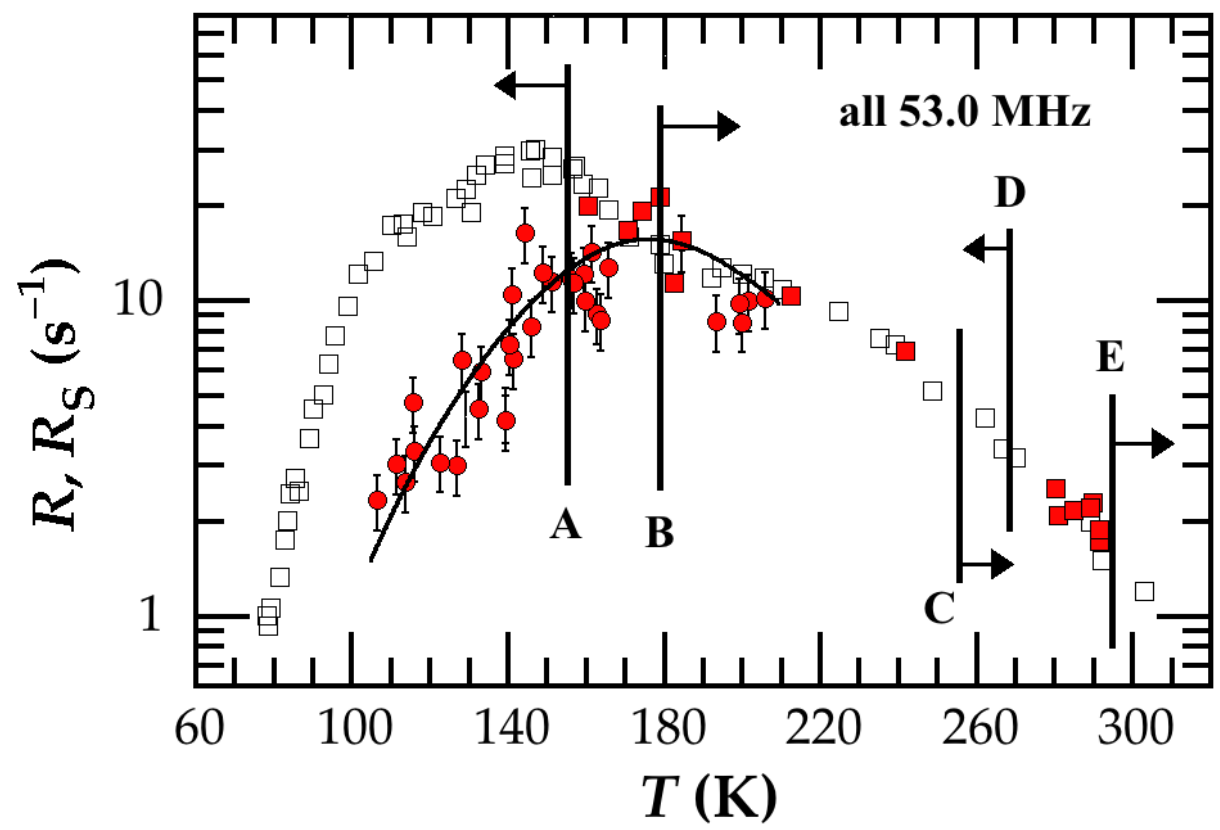

Fig. 9. Various ${ }^{1} \mathrm{H}$ spin-lattice relaxation rates versus temperature $T$ in 1,3,5-tri-t-butylbenzene (TTB) at an NMR frequency of $53.0 \mathrm{MHz} . R_{\mathrm{S}}[(\mathrm{red})]$ is the initial ${ }^{1} \mathrm{H}$ spin-lattice relaxation rate in a nonexponential relaxation process and $R[\square$ (green)] is the relaxation rate in an exponential relaxation process. The samples had been at room temperature for days or weeks (thermal preparation 3) or had been at $77 \mathrm{~K}$ for many hours (thermal preparation 4). In both cases, the sample was then inserted into the NMR coil that was preset at a temperature between 105 and 140 $\mathrm{K}$. Temperature was increased for subsequent measurements during a single day's experiments. Five days of experiments [three with thermal preparation 3 and two with thermal preparation 4 (one in liquid nitrogen for 12 hours and the other for 50 hours)] over a period of many years are represented here. The fit through the $R_{\mathrm{S}}$ data between 105 and $200 \mathrm{~K}$ is discussed in Section 2.7. The exponential relaxation rate $R$ [ $\square$ (empty squares)] in thrice-cycled samples (thermal preparation 1) shown in Fig. 6 is repeated here for comparison. The solid-solid phase transitions labeled by A through E seen (depending on thermal history) by scanning differential calorimetry (Fig. 4) are indicated by the black vertical lines with a right-pointing arrow meaning on heating and a left-pointing arrow meaning on cooling. 